\title{
THE INITIAL-VALUE PROBLEM FOR THE FORCED KORTEWEG-DE VRIES EQUATION
}

By

Jerry L. Bona

and

Bing-Yu Zhang

IMA Preprint Series \# 1253

September 1994

INSTITUTE FOR MATHEMATICS AND ITS APPLICATIONS

UNIVERSITY OF MINNESOTA

514 Vincent Hall

206 Church Street S.E.

Minneapolis, Minnesota 55455 


\title{
The Initial - Value Problem for the Forced Korteweg-de Vries Equation*
}

\author{
Jerry L. Bona \\ Department of Mathematics and Applied Research Laboratory \\ The Pennsylvania State University \\ University Park, PA 16802 \\ Bing-Yu Zhang \\ Department of Mathematical Sciences \\ University of Cincinnati \\ Cincinnati, Ohio 45221-0025
}

\begin{abstract}
The initial-value problem for the Korteweg-de Vries equation with a forcing term has recently gained prominence as a model for a number of interesting physical situations. At the same time, the modern theory for the initial-value problem for the unforced Korteweg-de Vries equation has taken great strides forward. The mathematical theory pertaining to the forced equation has not kept up with recent advances. This aspect is rectified here with the development of a broader theory for the initial-value problem for the forced Korteweg-de Vries equation. The results obtained include analytic dependence of solutions on both the initial condition and the forcing and allows the external forcing to lie in function classes sufficiently large that a Dirac $\delta$-function or its derivative is included.
\end{abstract}

${ }^{*}$ This work was supported in part by the National Science Foundation and the W. M. Keck Foundation. The paper was completed while the second author held a post-doctoral position in industrial mathematics at the Institute for Mathematics and its Applications at the University of Minnesota. The authors thank G. Ponce for advice and encouragement at the outset of the present study. 


\section{Introduction}

Considered herein is the initial-value problem (IVP) for the forced Korteweg-de Vries $(\mathrm{KdV})$ equation

$$
\left\{\begin{array}{l}
\partial_{t} u+u \partial_{x} u+\partial_{x}^{3} u=f(x, t), \\
u(x, 0)=u_{0}(x),
\end{array}\right.
$$

for $x, t \in R$. Here, the dependent variable $u=u(x, t)$ is a real-valued function of the independent variables $x$ and $t$ that in most situations where the equation appears as a model, correspond to distance measured in the direction of the waves propagation and elapsed time. The initial value $u_{0}$ and external forcing $f$ will be suitably restricted presently.

The problem (1.1) arises naturally in situations close to those that lead to the Korteweg-de Vries equation as an approximate model, but which feature suitably small non-homogeneities. For example, the IVP (1.1) has arisen in studying wave motion over a flat, horizontal bottom that has a localized perturbation (see Cole [9] or Grimshaw and Smyth [14]) and in attempting to determine the response in a channel to a surface disturbance moving into undisturbed fluid (cf. Akylas [1], Lee [23] and $\mathrm{Wu}[28])$. The forcing term $f$ may also be thought of as providing a rough accounting of terms that are neglected in arriving at the tidy $\mathrm{KdV}$ equation (1.2) below.

Interest in (1.1) lagged behind that associated with the IVP

$$
\left\{\begin{array}{l}
\partial_{t} u+u \partial_{x} u+\partial_{x}^{3} u=0, \quad x, t \in R \\
u(x, 0)=u_{0}(x), \quad x \in R
\end{array}\right.
$$

for the KdV equation. The intensive investigation of (1.2) by an army of scientists was sparked in large measure by the inverse-scattering theory pertaining thereto. So far, no effective means has been found to bring this fruitful theory to bear upon the forced $\mathrm{KdV}$ equation (1.1). Indeed, this has remained a major open problem since the 1970's (see Miura [24]).

The well-posedness of the IVP (1.2) in the classical, $L_{2}$-based Sobolev spaces $H^{s}(R)$ for $s>3 / 2$ was well established in the mid-1970's (cf. Bona and Smith [3], Bona and Scott [4], Saut and Temam [26], Kato [15], [16] and the references contained therein). In the early 1980's Kato [17] discovered a subtle and rather general smoothing effect for the IVP (1.2). While this effect had been apparent in the early work of Cohen [8] (see also Sachs [25]), the simplicity and power of his 
observations inspired new consideration of the IVP (1.2) by Constantin and Saut [10], Ginibre and Velo [12] and Kenig, Ponce and Vega [18], [19] for example. This theory shows the IVP (1.2) to be locally (resp. globally) well-posed in $H^{s}$ provided only that $s>3 / 4$ (resp. $s \geq 1$ ) [19], [20]. Recently, Bourgain [7] demonstrated (1.2) to be globally well posed in $H^{0}(R)=L^{2}(R)$ using a contraction-mapping argument in a very cleverly chosen space. Combining Bourgain's theory with their estimates in [20], Kenig, Ponce and Vega [21] showed shortly thereafter that (1.2) is locally well-posed in the space $H^{s}(R)$ provided only that $s>-5 / 8$.

By contrast, the theory pertaining to the IVP (1.1) for the forced KdV equation has remained less developed. The following result was given by Bona and Smith [3] in the early 1970's.

Theorem 1.1 For given $T>0$ and $s \geq 3$, if (i) $u_{0} \in H^{s}(R)$, (ii) $f \in C\left(-T, T ; H^{s}(R)\right.$ ), and (iii) $f_{t} \in C\left(-T, T ; L^{2}(R)\right)$, then the IVP (1.1) has a unique solution $u \in$ $C\left(-T, T ; H^{s}(R)\right) \cap C^{1}\left(-T, T ; L^{2}(R)\right)$. In addition, the solution u depends continuously in $C\left(-T, T ; H^{s}(R)\right)$ on $u_{0}$ in $H^{s}(R)$ and $f$ in $C\left(-T, T ; H^{s}(R)\right) \cap C^{1}\left(-T, T ; L^{2}(R)\right)$.

This result was strengthened recently by Zhang in [29] where he showed that the conclusion of Theorem 1.1 holds without assumption (iii).

It is our purpose here to bring the theory for the IVP (1.1) into the general range of what is known for the IVP (1.2). Four aspects of the IVP (1.1) will occupy us in the body of the paper. Use will be made throughout of the recent developments for the unforced problem (1.2) (cf. [6], [7], [12], [13], [18], [19], [20], [21], [30], [31]). While in most aspects it is only required to adapt the tools available in previous works, the theory that emerges is very much more satisfactory than the earlier results quoted above.

Before going into a little more detail, it is convenient to discuss briefly our notational conventions.

\section{Notation}

In general, if $X$ is a Banach space of functions of one or two variables, its norm will be denoted by $\|\cdot\|_{X}$ except for the abbreviations listed now. The norm for $L^{2}(R)$ will be written without decoration as simply $\|\cdot\|$ and the standard norm

$$
\|g\|_{s}^{2}=\int_{-T}^{T}\left(1+|\xi|^{2}\right)^{s}|\hat{g}(\xi)|^{2} d \xi
$$

for the $L^{2}$-based Sobolev space $H^{s}(R), s$ any fixed real number, will be written as indicated in (1.3). Here and elsewhere, a circumflex adorning a function of one or 
two real variables denotes that function's Fourier transform. In one instance, it will be useful to consider the Fourier transform of a function $g=g(x, t)$ in only the first variable $x$, and this will be indicated by the non-standard notation $\tilde{g}=\tilde{g}(\xi, t)$. As this partial transform only appears briefly, its notation should not cause confusion. If $X$ is a Banach space, $C(a, b ; X)$ is the functions $u:[a, b] \rightarrow X$ which are continuous. This is a Banach space with the norm

$$
\sup _{a \leq t \leq b}\|u(t)\|_{X}
$$

In case $a=-\infty$ or $b=\infty$, we will append a subscript $b$ to connote that the mappings $u$ are bounded. Thus $C_{b}(R ; X)$ is the space of bounded, continuous mappings of $R$ into $X$ equipped with the norm just displayed. The collection $L^{p}(a, b ; X)$ is defined similarly, as are the Sobolev classes $W^{k, p}(a, b ; X)$ of function whose first $k$ derivatives lie in $L^{p}(a, b ; X)$.

I. Well-posedness of the IVP (1.1) in the space $H^{s}(R)$

Our goal is to update the known results for the IVP (1.1) to the general level of Kenig, Ponce and Vega's recent work [21] on the IVP (1.2). Indeed, armed with the new tools introduced by Kenig, Ponce and Vega [18], [21] and Bourgain [7], we are able to show that

for given $T>0$ and $s>-5 / 8$, the IVP (1.1) is locally well-posed for initial data $u_{0}$ in the space $H^{s}(R)$ and forcing $f \in L^{2}\left(-T, T ; H^{s}(R)\right)\left(f \in L^{1}\left(-T, T ; H^{s}(R)\right)\right.$ if $s>3 / 4$ ).

As a consequence of the above well-posedness result, the IVP (1.1) establishes a nonlinear map $K_{I}$ from the space $H^{s}(R) \times L^{2}\left(-T, T ; H^{s}(R)\right)$ to the space $C\left(-T, T ; H^{s}(R)\right)$ by the specification $K_{I}\left(u_{0}, f\right):=u$, where $u$ is the solution of the IVP (1.1) corresponding to the initial data $u_{0}$ and the forcing function $f$.

\section{Regularity of the map $K_{I}$.}

For the homogeneous KdV equation, the IVP (1.2) also defines a nonlinear map $K_{H}$ from the space $H^{s}(R)$ to $C\left(-T, T ; H^{s}(R)\right)$. Bona and Smith [3], and Kato [15], [16] showed that $K_{H}$ is continuous from the space $H^{s}(R)$ to the space $C\left(-T, T ; H^{s}(R)\right)$. Then, Saut and Temam [26] proved that $K_{H}$ is Hölder continuous with exponent $\frac{1}{2}$ from the space $H^{s+1 / 2}(R)$ to the space $L^{\infty}\left(-T, T ; H^{s}(R)\right)$. These early results did not use smoothing properties of the equation. Much stronger regularity can be established by taking advantage of the various smoothing properties possessed by the $\mathrm{KdV}$ equation. Simply as a by-product of their contraction-principle approach to the IVP 
(1.2), Kenig, Ponce and Vega [20] showed that the map $K_{H}$ is Lipschitz continuous from the space $H^{s}(R)$ to the space $C\left(-T, T ; H^{s}(R)\right)$. Zhang [30] then proved that the map $K_{H}$ is infinitely many times Fréchet differentiable from the space $H^{s}(R)$ to the space $C\left(-T, T ; H^{s}(R)\right)$ and that for $\delta>0$ sufficiently small, the formal Taylor series expansion

$$
K_{H}(\phi+h)=\sum_{n=0}^{\infty} \frac{K_{H}^{(n)}(\phi)\left[h^{n}\right]}{n !}
$$

converges in $C\left(-T, T ; H^{s}(R)\right)$ uniformly for $\|h\|_{s} \leq \delta$, which is the same as saying that the map $K_{H}$ is analytic from the space $H^{s}(R)$ to the space $C\left(-T, T ; H^{s}(R)\right)$. Here, $K_{H}^{(n)}(\phi)$ is the $\mathrm{n}$-th derivative of $K_{H}$ at $\phi$, an n-multilinear map from the $\mathrm{n}$-fold product of $H^{s}(R)$ to $C\left(-T, T ; H^{s}(R)\right)$.

We show in this paper that

the map $K_{I}$ corresponding to the IVP (1.1) is analytic from the space $H^{s}(R) \times$ $L^{2}\left(-T, T ; H^{s}(R)\right)$ to the space $C\left(-T, T ; H^{s}(R)\right)$.

As a result, the solution $u$ of the IVP (1.1) can be expanded as a Taylor series with respect to its initial data $u_{0}$ and the forcing function $f$. Since each term in the Taylor series may be obtained by solving a linearized KdV equation, any solution of the nonlinear IVP (1.1) can be written as a series of solutions of associated linear problems.

\section{Smoothing properties of the IVP (1.1).}

It is a standard issue arising in the study of inhomogeneous partial differential equations to determine whether solutions have higher regularity than the forcing term. For the IVP (1.1), the regularity of solutions $u(x, t)$ in the spatial variable $x$ is usually the same as that of the forcing term $f(x, t)$. However, for the associated linear problem,

$$
\partial_{t} u+\partial_{x}^{3} u=f, \quad u(x, 0)=0,
$$

standard semigroup theory shows that the solution $u$ lies in $C\left(-T, T ; H^{s+3}(R)\right)$ if $f \in W^{1,1}\left(-T, T ; H^{s}(R)\right)$. The price paid for the extra spatial regularity is that $f$ is required to have stronger regularity in the temporal variable $t$. We present here similar results for the nonlinear problem (1.1). Indeed, we shall be able to prove the following sort of theorem, stated here with zero initial data to keep the statement simple.

For $u_{0}=0$ and $s>-5 / 8$, if the forcing term $f \in W^{1,2}\left(-T, T ; H^{s}(R)\right)$, then the solution $u \in C\left(-T, T ; H^{s+3}(R)\right)$ and if $s>-17 / 8$, then $f \in W^{\frac{1}{2}, 2}\left(-T, T ; H^{s}(R)\right)$ implies that $u \in C\left(-T, T ; H^{s^{\prime}}(R)\right)$ for any $s^{\prime}<s+3 / 2$. 
As a particular example, the theory allows one to take a Dirac $\delta$-function (or even the derivative of a $\delta$-function) as a forcing function acting on the right-hand side of the $\mathrm{KdV}$ equation and conclude the corresponding solution $u$ lies in $C\left(-T, T ; H^{s}(R)\right)$ for any $s<5 / 2$ (for any $s<0$ ).

Smoothing properties of the IVP (1.1) with respect to its initial data are also considered. The IVP (1.1) is shown to have the same smoothing properties as those proved by Kenig, Ponce and Vega [19] for the IVP (1.2).

IV. Global existence of solutions in the space $H^{s}(R)$.

It is familiar in nonlinear analysis that a global existence result for an initial-value problem usually follows from a local existence result and appropriate global a priori estimates. For the IVP (1.1), the needed global estimates when $s=0$ or $s \geq 1$ can be established with the aid of forced versions of the conservation laws appertaining to the unforced KdV equation (1.2). Consequently, we are able to show that for any $u_{0} \in H^{s}(R)$ and $f \in L^{2}\left(R ; H^{s}(R)\right)$, the corresponding solution of the IVP (1.1) exists globally in the space $H^{s}(R)$. However, when $0<s<1$ or $-5 / 8<s<0$, the needed estimates in $H^{s}(R)$ are not available. The question arises naturally as to whether a solution of the IVP (1.1) exists for all time or blows up in a finite time in the space $H^{s}(R)$ when $-5 / 8<s<0$ or $0<s<1$. This is an open question, even for the homogeneous IVP (1.2) (cf. [21]).

An interesting point that casts some light on this last mentioned issue follows from the analyticity of the map $K_{I}$. For any $s>-5 / 8$ and $T>0$, let $\mathcal{D}_{s}^{T}$ be the collection of all $\left(u_{0}, f\right) \in H^{s}(R) \times L^{2}\left(R, H^{s}(R)\right)$ for which the corresponding solution $u$ of the IVP (1.1) exists in the whole interval $(-T, T)$.

$$
\begin{aligned}
& \text { For }-5 / 8<s<0 \text { or } 0<s<1 \text { and any } T>0, \mathcal{D}_{s}^{T} \text { is a dense open subset of } \\
& H^{s}(R) \times L^{2}\left(R, H^{s}(R)\right) \text {. }
\end{aligned}
$$

The paper is organized as follows. In section 2, the most important linear estimates from Kenig, Ponce and Vega [21] are briefly reviewed. Then consideration is given to the associated linear IVP

$$
\left\{\begin{array}{l}
\partial_{t} u+\partial_{x}(v u)+\partial_{x}^{3} u=f, \\
u(x, 0)=u_{0}(x),
\end{array}\right.
$$

for $x, t \in R$ where $v=v(x, t)$ is a given function. The well-posedness of the IVP (1.5) in the space $H^{s}(R)$ is established and estimates of the solution in term of $u_{0}$ and $f$ are provided. This theory is the basis for the demonstration of analyticity of the map $K_{I}$. In section 3 , the well-posedness of the IVP $(1.1)$ in the space $H^{s}(R)$ 
$(s>-5 / 8)$ and the analyticity of the map $K_{I}$ are established. Instead of dealing directly with the nonlinear system (1.1) itself, we first consider the infinite system of linear equations

$$
\left\{\begin{array}{l}
\partial_{t} y_{1}+\partial_{x}\left(u y_{1}\right)+\partial_{x}^{3} y_{1}=h_{f} \\
y_{1}(x, 0)=h_{u_{0}}
\end{array}\right.
$$

and, for $n \geq 2$,

$$
\left\{\begin{array}{l}
\partial_{t} y_{n}+\partial_{x}\left(u y_{n}\right)+\partial_{x}^{3} y_{n}=-\frac{1}{2} \sum_{k=0}^{n-1}\left(\begin{array}{l}
n \\
k
\end{array}\right) \partial_{x}\left(y_{k} y_{n-k}\right), \\
y_{n}(x, 0)=0
\end{array}\right.
$$

where $u$ is assumed to be a solution of the IVP (1.1) corresponding to the initial data. $u_{0}$ and the forcing term $f$. According to the linear theory established in section 2, the linear system (1.6)-(1.7) is solvable. It is then shown that

$$
v=u+\sum_{k=1}^{\infty} \frac{y_{k}}{k !}
$$

is a solution of the IVP (1.1) corresponding to the initial data $u_{0}+h_{u_{0}}$ and the forcing function $f+h_{f}$ provided the size of $h_{u_{0}}$ and $h_{f}$ is small in a particular sense. The well-posedness the IVP (1.1) and the analyticity of the map $K_{I}$ follow as corollaries. In section 4 , we discuss smoothing properties of the system (1.1), while section 5 provides global existence of solutions of the IVP $(1.1)$ in $H^{s}(R)$-spaces. In section 6 , theory is developed for the periodic IVP for the forced KdV equation, namely (1.1) where $u_{0}$ is chosen from appropriate classes of periodic functions. Results similar to those established for the IVP (1.1) posed on the entire real line $R$ are obtained.

\section{Linear Estimates}

To begin, we introduce a special Sobolev-type space used by Kenig, Ponce and Vega in [21], which is a modified version of the space first introduced by Bourgain in $[7]$.

For any $s, b \in R$, let $Y_{s, b}$ be the completion of the space $S\left(R^{2}\right)$ of tempered test functions with respect to the norm

$$
\|f\|_{Y_{s, b}}^{2}=\int_{-\infty}^{\infty} \int_{-\infty}^{\infty}\left(1+\left|\tau-\xi^{3}\right|\right)^{2 b}(1+|\xi|)^{2 s}|\hat{f}(\xi, \tau)|^{2} d \xi d \tau
$$


where $\hat{f}(\xi, \tau)$ denotes the Fourier transform of $f(x, t)$. As shown in [21], if $u \in Y_{s, b}$ with $s>-1$ and $b>1 / 2$, one has

$$
u \in C_{l o c}^{\alpha}\left(R ; L_{t}^{2}(R)\right)
$$

for any $\alpha \in(0,1+s)$, and consequently

$$
u \in L_{x, l o c}^{p}\left(R ; L_{t}^{2}(R)\right),
$$

for $1 \leq p \leq \infty$. The space $Y_{s, b}$ has a very useful property described in the following lemma taken from [21, Lemma 3.4].

Lemma 2.1 Let there be given $s>-5 / 8$ and $\sigma \in C_{0}^{\infty}(R)$ with its support in the interval $(-1,1)$. Then there exists a $\beta_{0} \in(1 / 2,1)$ such that for any $b \in\left(1 / 2, \beta_{0}\right)$, there is a $\theta_{0}>(2 b-1) / 2$ for which

$$
\left\|\sigma^{2}\left(\delta^{-1} t\right) \partial_{x}(u v)\right\|_{Y_{s, b-1}} \leq c \delta^{\theta_{0}}\|u\|_{Y_{s, b}}\|v\|_{Y_{s, b}}
$$

for any $u, v \in Y_{s, b}$ and $0<\delta<1$.

The following useful result is an immediate corollary.

Lemma 2.2 Let $s, \sigma$ and $b$ be as in Lemma 2.1. Then, for any $u, v \in Y_{s, b}$ and $T>0$, there is a $c_{1}=c_{1}(T)$ such that

$$
\left\|\sigma\left(T^{-1} t\right) \partial_{x}(u v)\right\|_{Y_{s, b-1}} \leq c_{1}\|u\|_{Y_{s, b}}\|v\|_{Y_{s, b}}
$$

The space $Y_{s, b}$ has a stronger topology than the the space $C_{b}\left(R ; H^{s}(R)\right)$ as the following lemma shows.

Lemma 2.3 Let $b>1 / 2$ and $s \in R$ be given. Then $Y_{s, b} \subset C_{b}\left(R ; H^{s}(R)\right)$ and there is a constant $c>0$ such that for any $f \in Y_{s, b}$,

$$
\sup _{t \in R}\|f(\cdot, t)\|_{s} \leq c\|f\|_{Y_{s, b}} .
$$

Proof: According to the Plancherel Theorem,

$$
\begin{aligned}
\sup _{t \in R}\|f\|_{s}^{2} & =\sup _{t \in R} \int_{-\infty}^{\infty}(1+|\xi|)^{2 s}|\tilde{f}(\xi, t)|^{2} d \xi \\
& \leq \int_{-\infty}^{\infty}(1+|\xi|)^{2 s} \sup _{t \in R}|\tilde{f}(\xi, t)|^{2} d \xi
\end{aligned}
$$


where $\tilde{f}$ connotes the Fourier transform of $f(x, t)$ with respect to the spatial variable $x$. Then, using the Riemann-Lebesgue lemma and the fact that $b>1 / 2$, it is found that

$$
\begin{aligned}
\sup _{t \in R}|\tilde{f}(\xi, t)|^{2} & \leq c\|\hat{f}(\xi, \cdot)\|_{L^{1}(R)}^{2} \\
& \leq c \int_{-\infty}^{\infty}\left(1+\left|\tau-\xi^{3}\right|\right)^{2 b}|\hat{f}(\xi, \tau)|^{2} d \tau
\end{aligned}
$$

from which one obtains

$$
\begin{aligned}
\sup _{t \in R}\|f\|_{s}^{2} & \leq c \int_{-\infty}^{\infty} \int_{-\infty}^{\infty}\left(1+\left|\tau-\xi^{3}\right|\right)^{2 b}(1+|\xi|)^{2 s}|\hat{f}(\xi, \tau)|^{2} d \xi d \tau \\
& =c\|f\|_{Y_{s, b}}^{2}
\end{aligned}
$$

The proof is complete.

Let $\{W(t)\}_{-\infty}^{+\infty}$ denote the unitary group generated by the operator

$$
\text { Af }=-f^{\prime \prime \prime}
$$

in the space $L^{2}(R)$. Then the solution of the linear IVP

$$
\left\{\begin{array}{l}
\partial_{t} v+\partial_{x}^{3} v=0 \\
v(x, 0)=v_{0}(x)
\end{array}\right.
$$

for $x, t \in R$, is given by $v(\cdot, t)=W(t) v_{0}(\cdot)$ and the solution of the inhomogeneous equation

$$
\left\{\begin{array}{l}
\partial_{t} v+\partial_{x}^{3} v=f(x, t), \\
v(x, 0)=0
\end{array}\right.
$$

in the same range of $x$ and $t$ is expressed as

$$
v(\cdot, t)=\int_{0}^{t} W(t-\tau) f(\cdot, \tau) d \tau
$$

Now suppose that $\psi \in C_{0}^{\infty}(R)$ with supp $\psi \subset(-1,1)$ and $\psi(x)=1$ for every $x \in[-1 / 2,1 / 2]$. The following result may also be found in [21].

Lemma 2.4 For given $s \in R$ and $b \in(1 / 2,1]$, there is a constant $c$ such that

$$
\left\|\psi\left(\delta^{-1} t\right) W(t) u_{0}\right\|_{Y_{s, b}} \leq c \delta^{(1-2 b) / 2}\left\|u_{0}\right\|_{s}
$$

and

$$
\left\|\psi\left(\delta^{-1} t\right) \int_{0}^{t} W(t-\tau) f(\tau) d \tau\right\|_{Y_{s, b}} \leq c \delta^{(1-2 b) / 2}\|f\|_{Y_{s, b-1}}
$$

for any $\delta \in(0,1)$. 
Remark 2.5 Combining (2.1) and (2.6), one has

$$
\left\|\psi\left(\delta^{-1} t\right) \int_{0}^{t} W(t-\tau) \sigma^{2}(\tau / \delta)\left(\partial_{x}(u v)\right)(\cdot, \tau) d \tau\right\|_{Y_{s, b}} \leq c \delta^{\theta_{0}}\|u\|_{Y_{s, b}}\|v\|_{Y_{s, b}} .
$$

This comprises a global smoothing property of the linear KdV equation.

Attention is now turned to the more general linear problem

$$
\left\{\begin{array}{l}
\partial_{t} u+\partial_{x}(v u)+\partial_{x}^{3} u=f \\
u(x, 0)=u_{0}(x)
\end{array}\right.
$$

for $x, t \in R$, where $v=v(x, t)$ is a given function. The following theorem provides a key tool for demonstrating analyticity of the map $K_{I}$ defined by the IVP (1.1).

Theorem 2.6 Suppose to be given $s>-5 / 8, T>0$, b as in Lemma 2.1 with $1 / 2<b \leq 1$ and $v \in Y_{s, b}$. Then for any $u_{0} \in H^{s}(R)$ and $f \in Y_{s, b-1}$, the IVP (2.8) has a unique solution $u \in C\left(-T, T ; H^{s}(R)\right)$ which is the restriction to $(-T, T)$ of a function $\bar{u} \in Y_{s, b}$ that satisfies the estimate

$$
\|\bar{u}\|_{Y_{s, b}} \leq c_{1}\left(\left\|u_{0}\right\|_{s}+\|f\|_{Y_{s, b-1}^{\prime}}\right)
$$

where $c_{1}=c_{1}\left(T,\|v\|_{Y_{s, b}}\right)$.

Proof: We attend first to the local existence of a solution using Kenig, Ponce and Vega's contraction-mapping argument [20]. To this end, rewrite (2.8) in its equivalent integral-equation form

$$
u(\cdot, t)=W(t) u_{0}(\cdot)+\int_{0}^{t} W(t-\tau) f(\cdot, \tau) d \tau-\int_{0}^{t} W(t-\tau) \partial_{x}(u v)(\cdot, \tau) d \tau .
$$

For the given auxiliary data $\left(u_{0}, f\right)$ and $\delta \in(0,1)$, define a map $\Gamma$ of the space $Y_{s, b}$ as follows;

$$
\begin{array}{r}
\Gamma(w)(\cdot)=\psi\left(\delta^{-1} t\right) W(t) u_{0}(\cdot)+\psi\left(\delta^{-1} t\right) \int_{0}^{t} W(t-\tau) f(\cdot, \tau) d \tau- \\
\psi\left(\delta^{-1} t\right) \int_{0}^{t} W(t-\tau) \sigma^{2}\left(\delta^{-1} \tau\right) \partial_{x}(v w)(\cdot, \tau) d \tau
\end{array}
$$

for any $w \in Y_{s, b}$, where $\sigma \in C_{0}^{\infty}(R)$ with $\sigma=1$ on the support of $\psi$, and the support of $\sigma \subset(-1,1)$. Using Lemma 2.1 and Lemma 2.3, it is inferred that

$$
\begin{aligned}
\|\Gamma(w)\|_{Y_{s, b}} & \leq c \delta^{(1-2 b) / 2}\left(\left\|u_{0}\right\|_{s}+\|f\|_{Y_{s, b-1}}\right)+c \delta^{(1-2 b) / 2}\left\|\sigma^{2}(t / \delta) \partial_{x}(v w)\right\|_{Y_{s, b-1}} \\
& \leq c \delta^{(1-2 b) / 2}\left(\left\|u_{0}\right\|_{s}+\|f\|_{Y_{s, b-1}}\right)+c \delta^{\theta_{0}+\frac{1-2 b}{2}}\|v\| Y_{s, b}\|w\|_{Y_{s, b}}
\end{aligned}
$$


Since $\theta_{0}>(2 b-1) / 2$, one may choose $\delta<1$ such that

$$
2 c \delta^{\theta_{0}+\frac{1-2 b}{2}}\|v\|_{Y_{s, b}} \leq 1 .
$$

Define the quantity $M$ to be

$$
M=2 c \delta^{(1-2 b) / 2}\left(\left\|u_{0}\right\|_{s}+\|f\|_{Y_{s, b-1}}\right),
$$

and let $H_{M}$ connote the ball of radius $M$ about zero in $Y_{s, b}$. In these cicumstances, it follows straightforwardly that for any $w \in H_{M}$,

$$
\|\Gamma(w)\|_{Y_{s, b}} \leq M .
$$

Because of the choice of $M$ and $\delta$, a similar set of estimates shows that

$$
\left\|\Gamma\left(w_{1}\right)-\Gamma\left(w_{2}\right)\right\|_{Y_{s, b}} \leq \frac{1}{2}\left\|w_{1}-w_{2}\right\|_{Y_{s, b}}
$$

for any $w_{1}, w_{2} \in H_{M}$. Hence $\Gamma$ is a contraction on $H_{M}$ if $\delta$ and $M$ are chosen according to (2.10) and (2.11). As a consequence, there exists a $u \in H_{M}$ such that

$$
\begin{aligned}
u(\cdot, t)= & \psi\left(\delta^{-1} t\right) W(t) u_{0}(\cdot)+\psi\left(\delta^{-1} t\right) \int_{0}^{t} W(t-\tau) f(\cdot, \tau) d \tau \\
& -\psi\left(\delta^{-1} t\right) \int_{0}^{t} W(t-\tau) \sigma^{2}\left(\delta^{-1} \tau\right) \partial_{x}(u v)(\cdot, \tau) d \tau .
\end{aligned}
$$

In particular, one has

$$
u(\cdot, t)=W(t) u_{0}(\cdot)+\int_{0}^{t} W(t-\tau) f(\cdot, \tau) d \tau-\int_{0}^{t} W(t-\tau) \partial_{x}(u v)(\cdot, \tau) d \tau
$$

for $-\delta / 2 \leq t \leq \delta / 2$. Hence $u(x, t)$ is a solution of $(2.8)$ for $-\delta / 2 \leq t \leq \delta / 2$ and in this range of $t$, satisfies

$$
\|u(\cdot, t)\|_{Y_{s, b}} \leq 2 c \delta^{(1-2 b) / 2}\left(\left\|u_{0}\right\|_{s}+\|f\|_{Y_{s, b-1}}\right) .
$$

Because the local solution is obtained via the contraction-mapping principle, it is straightforwardly inferred to be unique within its function class.

This local solution is easily extended to the entire interval $(-T, T)$. Indeed, because the time of existence $\delta$ depends only on $v$ and the solution $u$ possesses the bound (2.12) in $Y_{s, b}$, a straightforward iteration of the contraction-mapping argument starting with $u\left(\cdot, t^{\prime}\right)$ as initial data at successively larger values of $\left|t^{\prime}\right|$ allows one to conclude in a finite number of steps existence and uniqueness on $(-T, T)$. The function $u$ obtained by pasting these local solutions together plainly lies in $C\left(-T, T ; H^{s}(R)\right)$. That 
it is the restriction to $(-T, T)$ of a function in $Y_{s, b}$ follows by writing it as a finite sum of elements of $Y_{s, b}$ using a partition of unity of $[-T, T]$ whose supports are based on the local patches of solution obtained via the contraction-mapping principle. The bound in (2.9) is obtained by applying (2.12) to each summand in the partition-ofunity representation of $u$. The proof is complete.

For any given $T>0, s \in R$ and $b>1 / 2$, define

$$
w^{*}=\left\{v \in Y_{s, b}: v(\cdot, t)=w(\cdot, t), \forall t \in(-T, T)\right\}
$$

and

$$
Y_{s, b}^{T}=\left\{w^{*}, w \in Y_{s, b}\right\} .
$$

The space $Y_{s, b}^{T}$ is a Banach space equipped with the quotient norm

$$
\left\|w^{*}\right\|_{Y_{s, b}^{T}}:=\inf _{v \in w^{*}}\|v\|_{Y_{s, b}} .
$$

According to the definition, $v \in Y_{s, b}^{T}$ is a family of elements in the space $Y_{s, b}$. It will occasionally be convenient to ignore the distinction between the equivalence class $v \in Y_{s, b}^{T}$ and a representative of this class. As long as values of $t \in(-T, T)$ are in question, this abuse causes no difficulty.

Remark 2.7 In the above notation, and keeping in mind the convention concerning equivalence classes and their representatives, Theorem 2.6 may be restated as follows.

Let $s>-5 / 8, T>0$ and $v \in Y_{s, b}$. Let $b$ in the range $(1 / 2,1]$ be chosen as in Lemma 2.1. Then for any $u_{0} \in H^{s}(R)$ and $f \in Y_{s, b-1}$, there is a unique $u \in Y_{s, b}^{T}$ which is a solution of (2.8) in the time interval $(-T, T)$. Moreover, one has

$$
\|u\|_{Y_{s, b}^{T}} \leq c_{1}\left(\left\|u_{0}\right\|_{s}+\|f\|_{Y_{s, b-1}}\right),
$$

where $c_{1}=c_{1}\left(T,\|v\|_{Y_{s, b}}\right)$ may tend to $+\infty$ if $T \rightarrow+\infty$ or $\|v\|_{Y_{s, b}} \rightarrow+\infty$.

Corollary 2.8 If $u_{0}=0$ and $f=\partial_{x}\left(g_{1} g_{2}\right)$ with $g_{1}, g_{2} \in Y_{s, b}$, then the estimate (2.13) can be written as

$$
\|u\|_{Y_{s, b}^{T}} \leq c_{2}\left\|g_{1}\right\|_{Y_{s, b}}\left\|g_{2}\right\|_{Y_{s, b}}
$$

where $c_{2}=c_{2}\left(T,\|v\|_{Y_{s, b}}\right)$ tends to zero as $T \rightarrow 0$.

Proof: Suppose that $T=\delta / 2$ where $\delta$ is determined by (2.10). Then from the first part of the proof of Theorem 2.6,

$$
\begin{aligned}
\|u\|_{Y_{s, b}^{T}} & \left.\leq 2 c_{1} \delta^{(1-2 b) / 2} \| \sigma^{2}\left(\delta^{-1} t\right) \partial_{x}\left(g_{1} g_{2}\right)\right) \|_{Y_{s, b-1}} \\
& \leq 2 \tilde{c_{1}} \delta^{\theta_{0}+(1-2 b) / 2}\left\|g_{1}\right\|_{Y_{s, b}}\left\|g_{2}\right\|_{Y_{s, b}} \\
& :=c_{2}\left\|g_{1}\right\| Y_{Y_{s, b}}\left\|g_{2}\right\|_{Y_{s, b}} .
\end{aligned}
$$


The case of an arbitrary finite value follows from this estimate and a partition-of-unity representation. The proof is complete.

\section{Well-posedness and Analyticity}

Throughout this section it is assumed that $s>-5 / 8$ and $b>1 / 2$ are as in Lemma 2.1. Define the product space $X_{s, b}$ to be

$$
X_{s, b}:=H^{s}(R) \times Y_{s, b-1}
$$

It follows from Theorem 2.6 that for given $T>0$ and $\left(u_{0}, f\right) \in X_{s, b}$, there corresponds at most one $u \in Y_{s, b}^{T}$ which is a solution of the nonlinear IVP (1.1) in the time interval $(-T, T)$. Thus solving the IVP $(1.1)$ defines a map $K_{I}$ from $X_{s, b}$ to $Y_{s, b}^{T}$ given by $u=K_{I}(\phi)$, where $\phi=\left(u_{0}, f\right) \in X_{s, b}$ and $u$ is the corresponding solution of the IVP (1.1) if it exists.

Let $\mathcal{D}_{s}^{T}=\mathcal{D}_{s}^{T}\left(K_{I}\right)$ denote the domain of the map $K_{I}$ in the space $X_{s, b}$. Obviously, $\mathcal{D}_{s}^{T}$ is not empty since $0 \in \mathcal{D}_{s}^{T}$. We show that $\mathcal{D}_{s}^{T}$ is an open set in the space $X_{s, b}$ and that the nonlinear map $K_{I}$ is analytic from $\mathcal{D}_{s}^{T}$ to $Y_{s, b}^{T}$.

Formally, if $K_{I}$ is an analytic mapping from $\mathcal{D}_{s}^{T}$ to $Y_{s, b}^{T}$, then, for $n=0,1,2, \cdots$, its n-th order Fréchet derivative $K_{I}^{(n)}(\phi)$ at $\phi \in \mathcal{D}_{s}^{T}$ exists and is the symmetric, n-linear map from $X_{s, b}$ to $Y_{s, b}^{T}$ given as

$$
K_{I}^{(n)}(\phi)\left[h_{1}, \ldots, h_{n}\right]=\left\{\frac{\partial^{n}}{\partial \xi_{1} \ldots \partial \xi_{n}} K_{I}\left(\phi+\sum_{k=1}^{n} \xi_{k} h_{k}\right)\right\}_{0, \ldots, 0}
$$

for any $h_{1}, h_{2}, \ldots, h_{n} \in X_{s, b}$. The homogeneous polynomial $K_{I}^{(n)}(\phi)\left[h^{n}\right]$ of degree $n$ induced by $K_{I}^{(n)}(\phi)$, where $h^{n}=(h, h, \ldots, h)$ (n-components), is

$$
K_{I}^{(n)}(\phi)\left[h^{n}\right]=\left\{\frac{d^{n}}{d \xi^{n}} K_{I}(\phi+\xi h)\right\}_{\xi=0}
$$

for $h=\left(h_{u_{0}}, h_{f}\right) \in X_{s, b}$. If we define $y_{n}$ by

$$
y_{n}=K_{I}^{(n)}(\phi)\left[h^{n}\right]
$$

then it is formally ascertained that for $-T<t<T$,

$$
\left\{\begin{array}{l}
\partial_{t} y_{1}+\partial_{x}\left(u y_{1}\right)+\partial_{x}^{3} y_{1}=h_{f} \\
y_{1}(x, 0)=h_{u_{0}}
\end{array}\right.
$$


and

$$
\left\{\begin{array}{l}
\partial_{t} y_{n}+\partial_{x}\left(u y_{n}\right)+\partial_{x}^{3} y_{n}=-\frac{1}{2} \sum_{k=0}^{n-1}\left(\begin{array}{l}
n \\
k
\end{array}\right) \partial_{x}\left(y_{k} y_{n-k}\right) \\
y_{n}(x, 0)=0
\end{array}\right.
$$

for $n \geq 2$, where $u=K_{I}(\phi)$ and $h=\left(h_{u_{0}}, h_{f}\right) \in X_{s, b}$.

On the other hand, for any $\phi=\left(u_{0}, f\right) \in \mathcal{D}_{s}^{T}$, let $u=K_{I}(\phi)$ and consider solving the linear systems

$$
\left\{\begin{array}{l}
\partial_{t} y_{1}+\partial_{x}\left(u y_{1}\right)+\partial_{x}^{3} y_{1}=h_{\cdot f} \\
y_{1}(x, 0)=h_{u_{0}},
\end{array}\right.
$$

and

$$
\left\{\begin{array}{l}
\partial_{t} y_{n}+\partial_{x}\left(u y_{n}\right)+\partial_{x}^{3} y_{n}=-\frac{1}{2} \psi\left(\frac{t}{2 T}\right) \sum_{k=0}^{n-1}\left(\begin{array}{c}
n \\
k
\end{array}\right) \partial_{x}\left(y_{k} y_{n-k}\right), \\
y_{n}(x, 0)=0
\end{array}\right.
$$

for $n \geq 2$, where $h=\left(h_{u_{0}}, h_{f}\right) \in X_{s, b}$ and $\psi$ is as in the previous section. It follows from Theorem 2.6 that (3.3)-(3.4) defines a homogeneous polynomial of degree $n$ from $X_{s, b}$ to $Y_{s, b}^{T}$ as described by the following proposition.

Proposition 3.1 Let $T>0$ and $\phi \in \mathcal{D}_{s}^{T}=\mathcal{D}_{s}^{T}\left(K_{I}\right)$ be given and let $u=K_{I}(\phi)$. Then (3.3)-(3.4) defines a homogeneous polynomial $K_{I}^{(n)}(\phi)\left[h^{n}\right]$ of degree $n$ from $X_{s, b}$ to $Y_{s, b}^{T}$. Moreover, there exists a constant $c_{3}$ such that

$$
\left\|y_{n}\right\|_{Y_{s, b}^{T}} \leq c_{3}^{n} n !\|h\|_{X_{s, b}^{\prime}}^{n}
$$

for any $n \geq 2$, where $c_{3}=c_{3}\left(T,\|u\|_{Y_{s, b}^{T}}\right)$, and it may be that $c_{3} \rightarrow+\infty$ as $T \rightarrow+\infty$ or $\|u\|_{Y_{s, b}^{T}} \rightarrow+\infty$, but in any case $c_{3} \rightarrow 0$ if $T \rightarrow 0$.

Proof: The proof is a straightforward consequence of Lemma 2.1 - Lemma 2.4 (cf. [30, Prop. 3.3].

Define a Taylor polynomial $P_{n}(h)$ of degree $n$, for $h \in X_{s, b}$, by

$$
P_{n}(h)=\sum_{k=0}^{n} \frac{K_{I}^{(k)}(\phi)\left[h^{k}\right]}{k !}=K_{I}(\phi)+\sum_{k=1}^{n} \frac{y_{k}}{k !},
$$

and a Taylor series by

$$
P(h)=\sum_{k=0}^{\infty} \frac{K_{I}^{(k)}(\phi)\left[h^{k}\right]}{k !} .
$$


Proposition 3.2 For any $\phi=\left(u_{0}, f\right) \in \mathcal{D}_{s}^{T}=\mathcal{D}_{s}^{T}\left(K_{I}\right)$, there exists an $\eta>0$ depending only on $\left\|K_{I}(\phi)\right\|_{Y_{s, b}^{T}}$ such that the formal Taylor series (3.7) is uniformly convergent in the space $Y_{s, b}^{T}$ with respect to $h \in X_{s, b}$ with $\|h\|_{X_{s, b}} \leq \eta$. Moreover, if $v=P(h)$, then $v \in Y_{s, b}^{T}$ solves the IVP

$$
\left\{\begin{array}{l}
\partial_{t} v+v \partial_{x} v+\partial_{x}^{3} v=f+h_{f}, \\
v(x, 0)=u_{0}+h_{u_{0}}
\end{array}\right.
$$

for $-T \leq t \leq T$.

Proof: It is readily seen that the sequence $\left\{P_{n}(h)\right\}_{n=0}^{\infty}$ of Taylor polynomials is Cauchy in $Y_{s, b}^{T}$ uniformly for $h$ in the ball of radius $\eta$ in $X_{s, b}$ for suitable $\eta$. Indeed, because of Proposition 3.1, it transpires that, for $m \geq n \geq 0$,

$$
\begin{aligned}
\| P_{n}(h) & -P_{m}(h)\left\|_{Y_{s, b}^{T}}=\right\| \sum_{k=n}^{m} \frac{y_{k}}{k !} \|_{Y_{s, b}^{T}} \\
& \leq \sum_{k=n}^{m} \frac{\left\|y_{k}\right\|_{Y_{s, b}^{T}}}{k !} \\
& \leq \sum_{k=n}^{m} c_{3}^{k}\|h\|_{X_{s, b}}^{k} .
\end{aligned}
$$

If $\eta$ is chosen so that

$$
\eta \leq 1 /\left(2 c_{3}\right)
$$

then for $h \in X_{s, b}$ with $\|h\|_{X_{s, b}} \leq \eta$, one has

$$
\left\|P_{n}(h)-P_{m}(h)\right\|_{Y_{s, b}^{T}} \leq \sum_{k=n}^{m} \frac{1}{2^{k}}
$$

which goes to zero uniformly as $n, m \rightarrow \infty$.

Since $\left\{P_{n}(h)\right\}_{n=0}^{\infty}$ is a Cauchy sequence in the space $Y_{s, b}^{T}$, it makes sense to define $v=P(h)$ as its limit as $n \rightarrow \infty$. Then $v \in Y_{s, b}^{T}$ and $v$ solves the IVP (3.8). To see this, note first that

$$
\begin{aligned}
v(x, 0) & =\sum_{k=0}^{\infty} \frac{y_{k}(x, 0)}{k !} \\
& =u(x, 0)+y_{1}(x, 0) \\
& =u_{0}(x)+h_{u_{0}}(x) .
\end{aligned}
$$


Moreover, since the series $P(h)$ is absolutely convergent in the space $Y_{s, b}^{T} \subset C\left(-T, T ; H^{s}(R)\right)$, it follows that

$$
\begin{aligned}
v^{2} & =\left(u+\sum_{k=1}^{\infty} \frac{y_{k}}{k !}\right)^{2} \\
& =u^{2}+2 \sum_{k=1}^{\infty} \frac{u y_{k}}{k !}+\left(\sum_{k=1}^{\infty} \frac{y_{k}}{k !}\right)^{2} \\
& =2\left(\frac{1}{2} u^{2}+\sum_{k=1}^{\infty} \frac{u y_{k}}{k !}+\frac{1}{2} \sum_{k=1}^{\infty} \frac{1}{k !} \sum_{n=0}^{k-1}\left(\begin{array}{c}
k \\
n
\end{array}\right) y_{n} y_{n-k}\right) .
\end{aligned}
$$

In consequence, we have

$$
\begin{aligned}
\partial_{t} v & +\frac{1}{2} \partial_{x}\left(v^{2}\right)+\partial_{x}^{3} v=\partial_{t} u+\sum_{k=1}^{\infty} \frac{\partial_{t} y_{k}}{k !}+\partial_{x}^{3} u+ \\
& +\sum_{k=1}^{\infty} \frac{\partial_{x}^{3} y_{k}}{k !}+\frac{1}{2} \partial_{x}\left(u^{2}\right)+\sum_{k=1}^{\infty}\left\{\frac{\partial_{x}\left(u y_{k}\right)}{k !}+\frac{1}{2 k !} \sum_{n=0}^{k-1}\left(\begin{array}{c}
k \\
n
\end{array}\right) \partial_{x}\left(y_{n} y_{n-k}\right)\right\} \\
= & \left(\partial_{t} u+\frac{1}{2} \partial_{x}\left(u^{2}\right)+\partial_{x}^{3} u\right)+\left(\partial_{t} y_{1}+\partial_{x}\left(u y_{1}\right)+\partial_{x}^{3} y_{1}\right)+ \\
+ & \sum_{k=2}^{\infty} \frac{1}{k !}\left\{\partial_{t} y_{k}+\partial_{x}\left(u y_{k}\right)+\frac{1}{2} \sum_{n=0}^{k-1}\left(\begin{array}{c}
k \\
n
\end{array}\right) \partial_{x}\left(y_{n} y_{n-k}\right)+\partial_{x}^{3} y_{k}\right\} \\
& =f+h_{f} .
\end{aligned}
$$

The proof is complete.

The following theorem is now readily adduced.

Theorem 3.3 (Analyticity) For any $T>0$, the IVP (1.1) establishes a map $K_{I}$ from the space $X_{s, b}$ to the space $Y_{s, b}^{T}$ having as its domain $\mathcal{D}_{s}^{T}=\mathcal{D}_{s}^{T}\left(K_{I}\right)$ a nonempty open subset of $X_{s, b}$. The map $K_{I}$ is analytic from $\mathcal{D}_{s}^{T}$ to $Y_{s, b}^{T}$ in the sense that for any $\phi \in \mathcal{D}_{s}^{T}$, there exists an $\eta>0$ such that for any $h \in X_{s, b}$ with $\|h\|_{X_{s, b}} \leq \eta$, the Taylor series expansion

$$
K_{I}(\phi+h)=\sum_{n=0}^{\infty} \frac{K_{I}^{(n)}(\phi)\left[h^{n}\right]}{n !}
$$

converges in the space $Y_{s, b}^{T}$. Moreover, the convergence is uniform with regard to $h$ in the aforementioned ball in $X_{s, b}$.

In particular, since $0 \in \mathcal{D}_{s}^{T}$, there exists an $\eta>0$ depending on $T$, such that for any $\phi=\left(u_{0}, f\right) \in X_{s, b}$ with $\|\phi\|_{X_{s, b}} \leq \eta$, the IVP (1.1) has a unique solution 
$u \in Y_{s, b}^{T}$ defined at least in the time interval $(-T, T)$. Moreover, according to (3.9) and Proposition 3.1, $\eta \rightarrow \infty$ as $T \rightarrow 0$. The local well-posedness of the IVP (1.1) thus follows as a corollary to Theorem 3.3.

Theorem 3.4 (Local well-posedness) For any $u_{0} \in H^{s}(R)$ and $f \in Y_{s, b-1}$, there exists a $T=T\left(\left\|u_{0}\right\|_{s},\|f\|_{Y_{s, b-1}}\right)$ and a unique $u \in Y_{s, b}^{T}$ which is a solution of the IVP (1.1) on the time interval $(-T, T)$ and which satisfies

$$
\|u\|_{Y_{s, b}} \leq c\left(\left\|u_{0}\right\|_{s}+\|f\|_{Y_{s, b-1}}\right)
$$

for some constant $c=c\left(\left\|u_{0}\right\|_{s},\|f\|_{Y_{s, b-1}}\right)>0$. Moreover, for any $T^{\prime}<T$, there exists a neighborhood $U$ of $\left(u_{0}, f\right)$ in the space $X_{s, b}$ such that the map $K_{I}$ is analytic from $U$ to $Y_{s, b}^{T^{\prime}}$.

\section{Smoothing Properties}

Consideration is first given to smoothing properties of the IVP (1.1) with regard to the forcing term $f$.

Lemma 4.1 For any $s, b \in R$, the embedding

$$
W^{1-b, 2}\left(R, H^{s-3(1-b)}(R)\right) \subset Y_{s, b-1}
$$

is continuous.

Proof: Since

$$
\begin{aligned}
1+|\xi| & =1+\left|\xi^{3}\right|^{1 / 3} \\
& \leq 1+\left(\left|\xi^{3}-\tau\right|+|\tau|\right)^{1 / 3} \\
& \leq c\left(1+\left|\xi^{3}-\tau\right|\right)^{1 / 3}(1+|\tau|)^{1 / 3}
\end{aligned}
$$

for some positive numerical constant $c$, it follows that, for any $u \in W^{1-b, 2}\left(R, H^{s-3(b-1)}(R)\right)$,

$$
\begin{aligned}
& \|u\|_{W^{1-b, 2}\left(R, H^{s-3(1-b)}(R)\right)}^{2}=\int_{-\infty}^{\infty} \int_{-\infty}^{\infty}(1+|\xi|)^{2 s}\left(1+\left|\tau-\xi^{3}\right|\right)^{2(b-1)}|u(\xi, \tau)|^{2} d \xi d \tau \\
& =\int_{-\infty}^{\infty} \int_{-\infty}^{\infty}(1+|\xi|)^{2(s-3(1-b))} \frac{(1+|\tau|)^{2(1-b)}(1+|\xi|)^{6(1-b)}}{(1+|\tau|)^{2(1-b)}\left(1+\left|\tau-\xi^{3}\right|\right)^{2(1-b)}}|u(\xi, \tau)|^{2} d \xi d \tau \\
& \leq c \int_{-\infty}^{\infty} \int_{-\infty}^{\infty}(1+|\xi|)^{2(s-3(1-b))}(1+|\tau|)^{2(1-b)}|u(\xi, \tau)|^{2} d \xi d \tau \\
& =c\|u\|_{Y_{s, b-1}}^{2} .
\end{aligned}
$$


The proof is complete.

The next theorem now follows directly from Theorem 3.4.

Theorem 4.2 Let $s>-5 / 8$ be given and $b>1 / 2$ be as in Lemma 2.1. Then for any $u_{0} \in H^{s}(R)$ and $f \in W^{1-b, 2}\left(R, H^{s-3(1-b)}(R)\right)$, there is a $T_{1}>0$ and a unique $u \in Y_{s, b}^{T_{1}}$ which defines a solution of the IVP (1.1) corresponding to the initial data $u_{0}$ and the forcing $f$.

Note that the $b$ in the above theorem can be chosen as close to $\frac{1}{2}$ as one likes. As a consequence, we have the following result.

Corollary 4.3 Let $s>-17 / 8$ be given. For any $f \in W^{\frac{1}{2}, 2}\left(R, H^{s}(R)\right)$ and $u_{0} \in$ $H^{s+\frac{3}{2}}(R)$, there exists a $T_{1}>0$ such that the corresponding solution $u$ of the IVP (1.1) lies in the space $C\left(-T_{1}, T_{1}, H^{s^{\prime}}(R)\right)$ for any $s^{\prime}<s+3 / 2$.

As Corollary 4.3 indicates, the price paid for a gain in spatial regularity of the solution of the IVP (1.1) is the assumption of more temporal regularity in the forcing function $f$. If one is willing to assume further temporal regularity, then the conclusion of the last result can be strengthened.

Theorem 4.4 Let $T>0$ and $s>-5 / 8$ be given and let $b>1 / 2$ be chosen as in Lemma 2.1. Then for any $u_{0} \in H^{s+3}(R)$ and $f \in W^{1,2}\left(-T, T ; H^{s}(R)\right)$, there exists a $T_{1}>0$ and a unique $u \in Y_{s, b}^{T_{1}}$ which is a solution of the IVP (1.1) over the time interval $\left(-T_{1}, T_{1}\right)$ where $T_{1} \leq T$ depends on $\left\|u_{0}\right\|_{s+3}$ and $\|f\|_{Y_{s, b-1}}$. Moreover,

$$
\partial_{t} u \in Y_{s, b}^{T} \quad \text { and } \quad u \in C\left(-T_{1}, T_{1} ; H^{s+3}(R)\right) \text {. }
$$

Proof: If $v=\partial_{t} u$, then $v$ is a solution of the IVP

$$
\left\{\begin{array}{l}
\partial_{t} v+\partial_{x}(u v)+\partial_{x}^{3} v=f_{t}, \\
v(x, 0)=f(x, 0)-u_{0}^{\prime \prime \prime}(x)-u_{0}(x) u_{0}^{\prime}(x) .
\end{array}\right.
$$

Because of the regularity of the initial data and the forcing term, it is deduced from the preceding theory that $u$ and $\partial_{t} u$ lie in $Y_{s, b}^{T_{1}}$ for some $T_{1}>0$. In Fourier transformed variables, this means

$$
\int_{-\infty}^{\infty} \int_{-\infty}^{\infty}(1+|\xi|)^{2 s}(1+|\tau|)^{2}\left(1+\left|\tau-\xi^{3}\right|\right)^{2 b}|\hat{u}(\xi, \tau)|^{2} d \xi d \tau<\infty
$$

Since $b<1$, there is a constant $c$ such that

$$
(1+|\xi|)^{6 b} \leq c\left(1+\left|\tau-\xi^{3}\right|\right)^{2 b}(1+|\tau|)^{2}
$$


Hence it follows that

$$
\begin{aligned}
& \int_{-\infty}^{\infty} \int_{-\infty}^{\infty}(1+|\xi|)^{2(s+3 b)}(1+|\tau|)^{1-b}|\hat{u}(\xi, \tau)|^{2} d \xi d \tau \\
& \quad \leq \int_{-\infty}^{\infty} \int_{-\infty}^{\infty}(1+|\xi|)^{2 s}(1+|\tau|)^{2}\left(1+\left|\tau-\xi^{3}\right|\right)^{2 b}|\hat{u}(\xi, \tau)|^{2} d \xi d \tau \\
& \quad<+\infty
\end{aligned}
$$

or, what is the same,

$$
u \in W^{1-b, 2}\left(R ; H^{s+3 b}(R)\right) .
$$

This in turn implies that

$$
\partial_{x} u \in L^{2}\left(-T_{1}, T_{1} ; H^{s+3 b-1}(R)\right)
$$

and

$$
u \partial_{x} u \in L^{2}\left(-T_{1}, T_{1} ; H^{s}(R)\right) .
$$

Finally, it is seen by writing the equation in the form

$$
u_{x x x}=f-u u_{x}-u_{t}
$$

that

$$
u \in C\left(-T_{1}, T_{1} ; H^{s+3}(R)\right)
$$

The proof is complete.

Remark 4.5 If it is only assumed $f, f_{t} \in Y_{s, b-1}$ rather than $f \in W^{1,2}\left(-T, T ; H^{s}(R)\right)$, we still have

$$
u \in W^{1-b, 2}\left(R: H^{s+3 b}(R)\right) .
$$

This follows immediately from the sort of calculation appearing in the proof of the last theorem.

Attention is now given to smoothing properties of the IVP (1.1) with regard to its initial data, which is the property that solutions may be more regular in their spatial variable than the initial data. Let $s>0$ and $T>0$ be given. For a function $w: R \times[-T, T] \rightarrow R$, define the quantities

$$
\begin{gathered}
\lambda_{1}(T, w)=\sup _{[-T, T]}\|w(\cdot, t)\|_{s} \\
\lambda_{2}(T, w)=\left(\sup _{x} \int_{-T}^{T}\left|D^{s} \partial_{x} w(x, t)\right|^{2} d t\right)^{1 / 2}
\end{gathered}
$$




$$
\lambda_{3}(T, w ; l)=\left(\int_{-T}^{T}\left\|J^{l} \partial_{x} w(\cdot, t)\right\|_{\infty}^{4} d t\right)^{1 / 4}
$$

with $l \in[0, s-3 / 4]$ where $J^{s}=\left(1-\partial_{x}^{2}\right)^{s / 2}$,

$$
\lambda_{4}(T, w ; r)=(1+T)^{-\rho}\left(\int_{R} \sup _{[-T, T]}\left|J^{r} w(x, t)\right|^{2} d x\right)^{1 / 2}
$$

with $r \in[0, s-3 / 4]$ and $\rho>3 / 4$ a fixed constant, and

$$
\Lambda_{l, r}^{s}(T ; w)=\max \left\{\lambda_{1}(T, w), \lambda_{2}(T, w), \lambda_{3}(T, w ; l), \lambda_{4}(T, w ; r)\right\} .
$$

Define also the function class $X_{r, l}^{T, s}$ by

$$
X_{r, l}^{T, s}=\left\{w \in C\left(-T, T ; H^{s}(R): \Lambda_{r, l}^{s}(T, w)<\infty\right\}\right.
$$

for $(r, l) \in\left[0, s-\frac{3}{4}\right] \times\left[0, s-\frac{3}{4}\right)$. This linear space is a Banach space when equipped with the norm

$$
\|w\|_{X_{r, l}^{T, s}}:=\Lambda_{r, l}^{s}(T, w)
$$

introduced by Kenig, Ponce and Vega [20]. Clearly, $X_{r, l}^{T, s}$ is a subspace of $C\left(-T, T ; H^{s}(R)\right)$ with a stronger topology. It has the following important properties established in [20].

Proposition 4.6 Let $T>0, s>3 / 4$ and $(l, r) \in[0,3 / 4] \times[0,3 / 4)$ be given. Then we have

$$
\begin{gathered}
\left\|W(t) u_{0}\right\|_{X_{r, l}^{T, s}} \leq c\left\|u_{0}\right\|_{s} \\
\left\|\int_{0}^{t} W(t-\tau) f(\cdot, \tau) d \tau\right\|_{X_{r, l}^{T, s}} \leq c(1+T)^{\rho} \int_{-T}^{T}\|f(\cdot, \tau)\|_{s} d \tau
\end{gathered}
$$

and

$$
\int_{-T}^{T}\left\|\partial_{x}(u v)\right\|_{s} d t \leq c T^{1 / 2}(1+T)^{\rho}\|u\|_{X_{0,0}^{T, s}}\|v\|_{X_{0,0}^{T, s}}
$$

where $\rho>3 / 4$ is constant.

Remark 4.7 The estimate (4.2) reveals a stronger version of both the Kato smoothing effect and the Strichartz' global smoothing effect for the unitary group $W(t)$ (cf. [20]). The estimates (4.3) and (4.4) show a global smoothing property which is similar to $(2.7)$.

In [20], Kenig, Ponce and Vega showed that for any $u_{0} \in H^{s}(R)$ with $s>3 / 4$, the IVP (1.2) for the homogeneous KdV equation has a unique solution $u \in X_{r, l}^{T, s}$. A similar result holds for the IVP (1.1) of the forced KdV equation. Indeed, the same arguments as those used in section 3 provide the following result. 
Theorem 4.8 Let $s>3 / 4, T>0$ and $(l, r) \in[0, s-3 / 4] \times[0, s-3 / 4)$ be given. Then for any $\left(u_{0}, f\right) \in H^{s}(R) \times L^{1}\left(-T, T ; H^{s}(R)\right)$, there is a

$$
T_{1}=T_{1}\left(\left\|u_{0}\right\|_{s},\|f\|_{L^{1}\left(-T, T ; H^{s}(R)\right)}\right) \leq T
$$

and a unique solution $u \in X_{r, l}^{T_{1}, s}$ to the IVP (1.1) satisfying

$$
\|u\|_{X_{r, l}^{T_{1}, s}} \leq c\left(\left\|u_{0}\right\|_{s}+\|f\|_{L^{1}\left(-T, T ; H^{s}(R)\right)}\right) .
$$

Moreover, for any $T^{\prime}<T_{1}$, there exists a neighborhood $U$ of $\left(u_{0}, f\right)$ in the space $H^{s}(R) \times L^{1}\left(-T, T ; H^{s}(R)\right)$ such that the map $K_{I}$ is analytic from $U$ to the space $X_{l, r}^{T^{\prime}, s}$.

The space $L^{2}\left(-T, T ; H^{s}(R)\right)$ is a subspace of the space $L^{1}\left(-T, T ; H^{s}(R)\right)$ and the space $Y_{s, b-1}$. But the space $Y_{s, b}^{T}$ and the space $X_{r, l}^{T, s}$ are not related by one being included in the other. Neither are the spaces $L^{1}\left(-T, T ; H^{s}(R)\right)$ and $Y_{s, b-1}$ so related. This raises an interesting question. Suppose $u_{0} \in H^{s}(R)$ and $f \in L^{2}\left(-T, T ; H^{s}(R)\right)$ with $s>3 / 4$. Then Theorem 3.4 provides a solution $u_{1} \in Y_{s, b}^{T}$ for the IVP (1.1) and Theorem 4.8 provides us another solution $u_{2} \in X_{r, l}^{T, s}$. Are these two solutions the same? This question is related to the following uniqueness problem for the IVP (1.1).

Uniqueness Problem: Suppose $u_{1}, u_{2} \in C\left(-T, T ; H^{s}(R)\right)$ are both solutions of the equation

$$
\partial_{t} u+u \partial_{x} u+\partial_{x}^{3} u=f
$$

for some $f \in L^{2}\left(-T, T, H^{s}(R)\right)$. Does the fact $u_{1}(\cdot, 0)=u_{2}(\cdot, 0)$ in the space $H^{s}(R)$ imply $u_{1}(\cdot, t) \equiv u_{2}(\cdot, t)$ in $H^{s}(R)$ for $t \in[-T, T]$ ?

The answer is affirmative if $s>3 / 2$ and the proof is an easy exercise. The issue remains open when $s \leq 3 / 2$. There are many discussions in the literature about the uniqueness problem for the IVP (1.2) for the KdV equation (cf. [13] and [22]). But the results thus far extant require that either the solution $u$ is in a stronger space than $C\left(-T, T ; H^{s}(R)\right)$ or the initial data decays at a certain rate as $x \rightarrow \pm \infty$. The uniqueness results appearing in Theorem 3.4 and Theorem 4.8 also require solutions in spaces stronger than $C\left(-T, T ; H^{s}(R)\right)$, namely $Y_{s, b}^{T}$ and $X_{r, l}^{T, s}$, respectively. On the other hand, these uniqueness results do imply uniqueness of the so-called strong solutions of the IVP (1.1). These are defined as follows.

Definition: A function $u \in C\left(-T, T ; H^{s}(R)\right)$ is called a strong solution of the IVP (1.1) if there exists a sequence $\left\{u_{m}\right\}_{m=1}^{\infty}$ lying in $C^{\infty}\left(-T, T ; H^{\infty}(R)\right)$ such that

$$
\partial_{t} u_{m}+u_{m} \partial_{x} u_{m}+\partial_{x}^{3} u_{m}=f_{m}
$$


for $x \in R, t \in(-T, T)$,

$$
\lim _{m \rightarrow \infty} \sup _{-T<t<T}\left\|u_{m}(\cdot, t)-u(\cdot, t)\right\|_{s}=0,
$$

and for which

$$
\lim _{m \rightarrow \infty}\left\|f_{m}-f\right\|_{L^{2}\left(-T, T ; H^{s}(R)\right)}=0 .
$$

Proposition 4.9 Let $s>-5 / 8$ and $T>0$ be given. Then the IVP (1.1) has at most one strong solution $u \in C\left(-T, T ; H^{s}(R)\right)$ for any given $u_{0} \in H^{s}(R)$ and $f \in$ $L^{2}\left(-T, T ; H^{s}(R)\right)$.

Proof: It suffices to show that if $u \in C\left(-T, T ; H^{s}(R)\right)$ is a strong solution of the IVP (1.1), then $u \in Y_{s, b}^{T}$ since the IVP (1.1) has at most one solution in this space.

Let $\left\{u_{m}\right\}_{m=1}^{\infty}$ be a sequence of functions corresponding to the assumption that $u$ is a strong solution of the IVP (1.1). Our theory shows that for all $m, u_{m} \in Y_{s, b}^{T}$. Note that $f_{m}$ tends to $f$ in the space $L^{2}\left(-T, T ; H^{s}(R)\right)$ and that $u_{m}(\cdot, 0)$ tends to $u_{0}(\cdot)$ in the space $H^{s}(R)$. In addition, $\sup _{-T<t<T}\left\|u_{m}(\cdot, t)\right\|_{s}$ is bounded independently of $m$. It follows from the local well-posedness result in Theorem 4.8 that the sequence $\left\{u_{m}\right\}_{m=1}^{\infty}$ is a Cauchy sequence in the space $Y_{s, b}^{T}$ whose limit $v$ is a solution of the IVP (1.1) corresponding to the given $u_{0}$ and $f$. Since $u$ is a limit of $\left\{u_{m}\right\}$ in the space $C\left(-T, T ; H^{s}(R)\right)$ and the space $Y_{s, b}^{T}$ is a stronger subspace of $C\left(-T, T ; H^{s}(R)\right)$, we must have $u \equiv v$.

Corollary 4.10 Let $s>3 / 4$ and $T>0$ be given. Let $u_{1}$ be the unique solution of the IVP (1.1) in the space $X_{r, l}^{T, s}$ and $u_{2}$ the unique solution of the IVP (1.1) in the space $Y_{s, b}^{T}$. Then $u_{1} \equiv u_{2}$ for $-T \leq t \leq T$.

Proof: It follows from Proposition 4.9 and the fact that both $u_{1}$ and $u_{2}$ are strong solutions of the IVP (1.1).

In consequence of these developments, we have the following result.

Theorem 4.11 Let $s>3 / 4$ and $T>0$ be given. For any $u_{0} \in H^{s}(R)$ and $f \in$ $L^{2}\left(-T, T ; H^{s}(R)\right)$, there exists a $T_{1}=T_{1}\left(\left\|u_{0}\right\|_{s},\|f\|_{L^{2}\left(-T, T ; H^{s}(R)\right)}\right) \leq T$ such that the IVP (1.1) has a unique strong solution $u \in C\left(-T_{1}, T_{1} ; H^{s}(R)\right)$. Moreover, the solution lies in $Y_{s, b}^{T_{1}}$ and $X_{l, r}^{T_{1}, s}$, where $b>1 / 2$ is as in Lemma 2.1 and $(l, r)$ is as in Proposition 4.6. 


\section{Global Existence Results in the Space $H^{s}(R)$}

The local well-posedness theory developed in the preceding sections leads naturally to consideration of whether or not the solutions can generally be continued in time, so becoming global solutions of the IVP. Of course, if the auxiliary data $\left(u_{0}, f\right)$ is sufficiently regular, global solutions are known to obtain therefrom via the earlier theory [3], [29]. However, for weaker specification, it is possible that solutions might blow up in finite time, so ceasing to exist at a certain point. This issue arises pointedly when one is concerned with the IVP (1.1) as an approximate description of wave phenomena.

The following two criteria for whether or not a solution of the IVP (1.1) ceases to exist in finite time follow from Theorem 3.4 and 4.8 , respectively, by a standard argument. Throughout this section, we continue to suppose that $b>1 / 2$ is fixed in accordance with the requirement that Lemma 2.1 is valid.

Proposition 5.1 Let $s>-5 / 8$ and $T>0$ be given. Then for any $u_{0} \in H^{s}(R)$ and $f \in Y_{s, b-1}$, there exists a maximal value $T_{1}$ with $0<T_{1} \leq T$ such that the IVP (1.1) has a unique solution $u \in Y_{s, b}^{T_{2}}$ for any $T_{2}<T_{1}$. The maximal value $T_{1}<T$ if and only if

$$
\lim _{t \rightarrow T_{1}^{-}}\|u(\cdot, t)\|_{s}=+\infty .
$$

Proposition 5.2 Let $s>3 / 4,(l, r) \in[0, s-3 / 4] \times[0, s-3 / 4)$ and $T>0$ be given. Then for any $\left(u_{0}, f\right) \in H^{s}(R) \times L^{1}\left(-T, T ; H^{s}(R)\right)$, there is a maximal value $T_{1}$ with $0<T_{1} \leq T$ such that for any $T_{2}<T_{1}$, the IVP (1.1) has a unique solution $u \in X_{l, r}^{T_{2}, s}$ and $T_{1}<T$ if and only if

$$
\lim _{t \rightarrow T_{1}^{-}}\|u(\cdot, t)\|_{s}=+\infty
$$

In consequence of these results, global a priori estimates for solutions of the IVP (1.1) in the space $H^{s}(R)$ suffice to infer the global well-posedness of the IVP (1.1). It is to the provision of such bounds that attention is now turned.

Lemma 5.3 Let there be given $T>0$ and $u \in C\left(-T, T ; H^{\infty}(R)\right)$ a solution of the IVP (1.1). Then the following inequalities appertain to $u$ :

$$
\begin{gathered}
\sup _{[-T, T]}\|u(\cdot, t)\| \leq c\left(\left\|u_{0}\right\|+\int_{-T}^{T}\|f(\cdot, t)\| d t\right), \\
\sup _{[-T, T]}\|u(\cdot, t)\|_{1} \leq c\left(\left\|u_{0}\right\|^{2}+\left\|u_{0}\right\|_{1}+\int_{-T}^{T}\|f(\cdot, t)\|_{1} d t\right),
\end{gathered}
$$


and for any $s>0$,

$$
\sup _{[-T, T]}\|u(\cdot, t)\|_{s} \leq c_{s} \exp \left(\int_{-T}^{T}\left\|\partial_{x} u(\cdot, \tau)\right\|_{\infty} d \tau\right)\left(\left\|u_{0}\right\|_{s}+\int_{-T}^{T}\|f(\cdot, t)\|_{s} d t\right),
$$

where the numerical constants $c$ and $c_{s}$ are independent of $u_{0}$ and $f$.

Proof: The proof of the estimates (5.1) and (5.2) is standard. The estimate (5.3) follows from the argument used in the proof of Lemma 3.2 in [19].

Theorem 5.4 (Global well-posedness) Let $s \geq 1, T>0$ and $(l, r) \in[0, s-3 / 4] \times$ $[0, s-3 / 4)$. Then, for any $\left(u_{0}, f\right) \in H^{s}(R) \times L^{1}\left(-T, T ; H^{s}(R)\right)$, the IVP (1.1) has a unique solution $u \in X_{r, l}^{T, s}$. Moreover, the corresponding map $K_{I}$ is analytic from $H^{s}(R) \times L^{1}\left(-T . T ; H^{s}(R)\right)$ to the space $X_{r, l}^{T, s}$.

Proof: This follows from the global a priori estimates (5.2), (5.3), Proposition 5.2 and Theorem 4.8 .

Notice that the estimates (5.1)-(5.3) for smooth solutions are given in terms of the $H^{s}(R)$-norm of the initial data and the $L^{1}\left(-T, T, H^{s}(R)\right)$ norm of the forcing function $f$. Of course, for any $T>0$, we know

$$
L^{2}\left(-T, T, H^{s}(R)\right) \subset L^{1}\left(-T, T ; H^{s}(R)\right)
$$

and

$$
L^{2}\left(-T, T ; H^{s}(R)\right) \subset Y_{s, b-1} .
$$

However, the spaces $L^{1}\left(-T, T ; H^{s}(R)\right)$ and $Y_{s, b-1}$ are not related to each other by inclusion.

Theorem 5.5 (Global well-posedness) Let $T>0$ be given.

(i) For any $\left(u_{0}, f\right) \in L^{2}(R) \times L^{2}\left(-T, T ; L^{2}(R)\right)$, the IVP (1.1) has a unique solution $u \in Y_{0, b}^{T}$ and the associated correspondence $K_{I}$ is an analytic mapping between these spaces.

(ii) For any $s \geq 1$ and $\left(u_{0}, f\right) \in H^{s}(R) \times L^{2}\left(-T, T ; H^{s}(R)\right)$, the IVP (1.1) has a unique solution $u \in Y_{s, b}^{T} \cap X_{r, l}^{T, s}$ and in this case also $K_{I}$ is an analytic mapping between these function classes.

Proof: In the case $s=0$, the result follows directly from the a priori estimates (5.1) and Proposition 5.1 by a standard argument. 
If $s \geq 1$, the IVP $(1.1)$ has a solution $u \in X_{r, l}^{T, s}$ according to Theorem 5.4. In particular,

$$
\sup _{[-T, T]}\|u(\cdot, t)\|_{s}<+\infty
$$

On the other hand, from Corollary 4.10,

$$
u(\cdot, t)=v(\cdot, t) \quad \text { in the space } H^{s}(R), \text { for } t \in\left(-T_{1}, T_{1}\right)
$$

where $v$ is the unique solution of the IVP $(1.1)$ in the space $Y_{s, b}^{T_{1}}$ corresponding to $\left(u_{0}, f\right)$ for some $T_{1}>0$. Because of the estimate (5.4) and Theorem 3.4, the solution $v$ can be extended to the whole interval $(-T, T)$ in such a way that $v \in Y_{s, b}^{T}$ and $(5.5)$ holds for $t \in(-T, T)$. The proof is complete. $\square$,

The cases where $0<s<1$ or $-5 / 8<s<0$ are interesting since the needed global a priori estimates are not available, although the IVP (1.1) has been shown to be locally well-posed for these ranges of $s$. This raises the question mentioned before of whether the corresponding solutions blow up in finite time or exist globally in the space $H^{s}(R)$.

As an application of the analyticity of the map $K_{I}$, a partial answer to this problem can be provided. For $\left(u_{0}, f\right) \in X_{s, b}$, the corresponding solution $u$ of the IVP $(1.1)$ exists globally in the space $H^{s}(R)$ if and only if

$$
\left(u_{0}, f\right) \in \mathcal{D}_{s}^{T}=\mathcal{D}_{s}^{T}\left(K_{I}\right), \quad \text { for any } T>0
$$

Now we know that if $-5 / 8<s \leq 0$,

$$
L^{2}(R) \times L^{2}\left(R ; L^{2}(R)\right) \subset \mathcal{D}_{s}^{T}
$$

and if $0<s \leq 1$,

$$
H^{1}(R) \times L^{2}\left(R, H^{1}(R)\right) \subset \mathcal{D}_{s}^{T}
$$

for any $T>0$. In addition, $\mathcal{D}_{s}^{T}$ is a non-empty open subset of $X_{s, b}$ according to Theorem 3.1. The following theorem is then obvious.

Theorem 5.6 Let $s>-5 / 8$ be given. Then, for any $T>0, \mathcal{D}_{s}^{T}=\mathcal{D}_{s}^{T}\left(K_{I}\right)$ is a dense open subset of the space $X_{s, b}$.

We close this section by giving still another global existence result.

Theorem 5.7 Let $T>0$ and $s \geq 0$ be given. For any

$$
u_{0} \in H^{s+3}(R) \quad \text { and } \quad f \in W^{1,2}\left(-T, T ; H^{s}(R)\right) \text {, }
$$


there exists a unique $u \in Y_{s, b}^{T}$ which is a solution of the IVP (1.1) in the time interval $(-T, T)$. Moreover,

$$
\partial_{t} u \in Y_{s, b}^{T} \quad \text { and } \quad u \in C\left(-T, T ; H^{s+3}(R)\right) .
$$

Proof: It follows from Theorem $5.5(s=0)$ and Theorem 2.6 that $u, u_{t} \in Y_{0, b}^{T}$. Then using the argument presented in the proof of Theorem 4.4, one has $u \in$ $L^{2}\left(-T, T ; H^{s+3}(R)\right)$. In particular,

$$
\int_{-T}^{T}\left\|\partial_{x} u(\cdot, t)\right\|_{L^{\infty}(R)} d t<\infty
$$

It then follows from (5.3) that

$$
\sup _{[-T, T]}\|u(\cdot, t)\|_{s}<\infty
$$

for any $s \geq 0$. As a result, $u \in Y_{s, b}^{T}$. Then Theorem 4.11 implies

$$
\partial_{t} u \in Y_{s, b}^{T}, \quad u \in C\left(-T, T ; H^{s+3}(R)\right)
$$

for any $T>0$. The proof is complete.

\section{Forced KdV Equation on a Periodic Domain}

In this final section of the paper, we analyze the periodic initial-value problem for the forced $\mathrm{KdV}$ equation. The situation in view assumes that both the initial data and the forcing function as regards its spatial variation are periodic of the same period, and focuses on solutions having the same periodicity in space. This problem is usually somewhat artificial as regards application to physically interesting situations. However, it frequently arises in numerical simulation where unbounded domains are hard to model and the relative simplicity of imposing periodic boundary conditions is very attractive. As a model of physical reality, the idea is usually that the initial disturbance and the forcing often take place far from boundaries, and hence the imposition of periodicity should not affect the solution significantly provided the period is large enough. According to the theory in [5], this approach is justified over certain time scales provided the initial data is suitably evanescent at $\pm \infty$.

Here, we treat the periodic IVP with a finite period, and leave aside the question of the relationship between the periodic IVP and the pure IVP. Because the period $\lambda$ is finite and the equation is written in a frame of reference having no linear transport term $\partial_{x} u$, a simple change of variables allows us to assume $\lambda=1$, and this convenient normalization will be adopted hereafter. 
Let $H^{s}(S)$ denote the real Sobolev space of order $s(s \geq 0)$ on the unit length circle $S$ in the plane. $H^{s}(S)$ may be characterized as the space of real 1-periodic functions $v$ whose Fourier series

$$
v(x) \sim \sum_{-\infty}^{\infty} v_{k} \exp (2 i \pi k x)
$$

is such that

$$
\|v\|_{s}=\left\{\sum_{-\infty}^{\infty}(1+|k|)^{2 s}\left|v_{k}\right|^{2}\right\}^{\frac{1}{2}}<+\infty .
$$

The left-hand side of (6.2) defines a Hilbert-space norm on the linear space $H^{s}(S)$. Let $D^{s}$ represent the fractional derivative of order $s$, so if $v$ has the Fourier series in (6.1), then

$$
D^{s} v \sim \sum_{-\infty}^{\infty} v_{k}|k|^{s} \exp (2 i \pi k x)
$$

We consider the IVP for the forced KdV equation, namely

$$
\partial_{t} u+u \partial_{x} u+\partial_{x}^{3} u=f, \quad u(x, 0)=u_{0}(x)
$$

for $x \in S, t \in R$.

The IVP (6.3) is first normalized by subtracting the mean-value of a putative solution. For any integrable, real-valued function $g$ defined on $S$, its mean value is denoted by $[g]$ and is given by

$$
[g]=\int_{S} g(x) d x .
$$

Let $u$ be a solution of the IVP $(6.3)$ and let $v=u-\left[u_{0}\right]$. Then $v$ is a solution of the IVP

$$
\left\{\begin{array}{l}
\partial_{t} v+\left[u_{0}\right] \partial_{x} v+v \partial_{x} v+\partial_{x}^{3} v=f, \\
v(x, 0)=u_{0}(x)-\left[u_{0}\right] .
\end{array}\right.
$$

It is straightforward to see that $[v(\cdot, t)] \equiv 0$ provided that $[f(\cdot, t)] \equiv 0$.

Let $s \geq 0$ and $\beta \in R$ be given. For $w: S \times R \rightarrow R$, define $\Lambda_{j}^{\beta}(w), j=1,2,3$, to be

$$
\begin{gathered}
\Lambda_{1}^{\beta}(w)=\left(\sum_{n=-\infty}^{\infty}(1+|n|)^{2 s} \int_{-\infty}^{\infty}\left(1+\left|\tau-n^{3}+\beta n\right|\right)|\hat{w}(n, \tau)|^{2} d \tau\right)^{1 / 2} \\
\Lambda_{2}^{\beta}(w)=\left(\sum_{n=-\infty}^{\infty}(1+|n|)^{2 s} \int_{-\infty}^{\infty} \frac{|\hat{w}(n, \tau)|^{2}}{1+\left|\tau-n^{3}+\beta n\right|} d \tau\right)^{1 / 2}
\end{gathered}
$$




$$
\Lambda_{3}^{\beta}(w)=\left(\sum_{n=-\infty}^{\infty}(1+|n|)^{2 s}\left[\int_{-\infty}^{\infty} \frac{|\hat{w}(n, \tau)|}{1+\left|\tau-n^{3}+\beta n\right|} d \tau\right]^{2}\right)^{1 / 2}
$$

Let

$$
Z_{\beta}^{s}=\left\{w \in L^{2}(S \times R): \Lambda_{1}^{\beta}(w)<\infty\right\},
$$

which is a Hilbert space equipped with the norm

$$
\|w\|_{Z_{\beta}^{s}}=\Lambda_{1}^{\beta}(w) .
$$

In addition, let

$$
F_{\beta}^{s}=\left\{f(x, t): x \in S, t \in R ; \Lambda_{2}^{\beta}(f)+\Lambda_{3}^{\beta}(f)<\infty\right\}
$$

with the norm

$$
\|f\|_{F_{\beta}^{s}}:=\Lambda_{2}^{\beta}(f)+\Lambda_{3}^{\beta}(f) .
$$

Obviously, we have

$$
L^{2}\left(R, H^{s}(S)\right) \subset F_{\beta}^{s}
$$

The space $Z_{\beta}^{s}$ was first introduced by Bourgain [7] to deal with the periodic IVP for the homogeneous KdV equation. It has the following properties.

Lemma 6.1 Let $s \geq 0, \beta \in R$ and let $u, v \in Z_{\beta}^{s}$ satisfy

$$
[u](t) \equiv 0, \quad[v](t) \equiv 0 .
$$

Then it follows that

$$
\left\|\psi\left(\delta^{-1} t\right) \partial_{x}(u v)\right\|_{F_{\beta}^{s}} \leq c \delta^{\frac{1}{12}}\|u\|_{Z_{\beta}^{s}}\|v\|_{Z_{\beta}^{s}}
$$

where $\psi$ is as in Lemma 2.4, an element of $C_{0}^{\infty}(R)$, supp $\psi \subset(-1,1)$ and $\psi \equiv 1$ on $[-1 / 2,1 / 2]$.

Remark 6.2 This is very minor modification of Lemma 7.41 and 7.42 in Bourgain [7]. A nice feature of the spaces $Z_{\beta}^{s}$ is that their structure absorbs the dispersion relation of the KdV equation. The estimate (6.6) reveals a very subtle smoothing effect in this context.

The following two technical lemmas may be found worked out in Bourgain [7] and Zhang [31]. 
Lemma 6.3 For any $h \in Z_{\beta}^{s}$,

$$
\left\|\psi\left(\delta^{-1} t\right) h\right\|_{Z_{\beta}^{s}} \leq c(b) \delta^{(1-2 b) / 2}\|h\|_{Z_{\beta}^{s}},
$$

for any $b$ with $1 / 2<b \leq 1$, where $\psi$ is as above. The constant $c(b)$ may tend to $+\infty$ as $b \rightarrow 1 / 2$.

Let $\left\{W_{\beta}(t)\right\}_{-\infty}^{\infty}$ denote the unitary group generated in $L^{2}(S)$ by the operator

$$
A_{\beta} f=-f^{\prime \prime \prime}-\beta f^{\prime}
$$

which is defined for any $f \in H^{3}(S)$.

Lemma 6.4 Let $\psi$ be as above, let $\beta \in R$ be fixed and let $u_{0} \in H^{s}(S)$ and $f \in F_{\beta}^{s}$, where $s \geq 0$. Then we have

$$
\begin{gathered}
\left\|\psi\left(\delta^{-1} t\right) W_{\beta}(t) u_{0}\right\|_{Z_{\beta}^{s}} \leq c\left\|u_{0}\right\|_{s}, \\
\left\|\psi\left(\delta^{-1} t\right) \int_{0}^{t} W_{\beta}(t-\tau) f(\tau) d \tau\right\|_{Z_{\beta}^{s}} \leq c \delta^{\frac{1-2 b}{2}}\|f\|_{F_{\beta}^{s}},
\end{gathered}
$$

for any $b>1 / 2$, where $c$ may depend on $b$. For any $T$ with $0<T \leq \infty$ and $f \in F_{\beta}^{s}$,

$$
\sup _{[-T, T]}\left\|\psi\left(\delta^{-1} t\right) \int_{0}^{t} W_{\beta}(t-\tau) f(\cdot, \tau) d \tau\right\|_{s} \leq c \Lambda_{3}^{\beta}(f) .
$$

Define another space $X_{\beta}^{s}$ by

$$
X_{\beta}^{s}=\left\{\left(u_{0}, f\right) \in H^{s}(S) \times F_{\beta}^{s}:\left[u_{0}\right]=\beta, \quad[f(\cdot, t)] \equiv 0\right\}
$$

and for any $T>0$ and $s \geq 0$,

$$
\mathcal{Z}_{\beta, T}^{s}:=Z_{\beta}^{s} \cap C\left(-T, T ; H^{s}(R)\right) .
$$

As in the non-periodic case, the IVP (6.3) defines a nonlinear map $K_{P}$ from the space $X_{\beta}^{s}$ to the space $\mathcal{Z}_{\beta, T}^{s}$ via the correspondence $K_{P}\left(\left(u_{0}, f\right)\right):=u$, where $u \in \mathcal{Z}_{\beta, T}^{s}$ is the solution of the IVP $(6.3)$ on the time interval $(-T, T)$ corresponding to the initial data $u_{0}$ and the forcing term $f$.

Let $\mathcal{D}_{s}^{T}\left(K_{P}\right)$ be the domain of the map $K_{P}$. Then an argument entirely similar to that used in the non-periodic case gives the following results.

Theorem 6.5 Let $s \geq 0, T>0$ and $\beta \in R$ be given. Then the following results hold. 
1. (Analyticity) $\mathcal{D}_{s}^{T}\left(K_{P}\right)$ is a non-empty, dense, open set in the space $X_{\beta}^{s}$ and the map $K_{P}$ is analytic from its domain to the space $\mathcal{Z}_{\beta, T}^{s}$ (cf. [31]).

2. (local Well-posedness) For any $\left(u_{0}, f\right) \in X_{\beta}^{s}$, there exists a $T_{1}=T_{1}\left(\|u\|_{s},\|f\|_{F_{\beta}^{s}}\right)$ with $0<T_{1} \leq T$ such that the $\operatorname{IVP}$ (6.3) has a unique solution $u \in \mathcal{Z}_{\beta, T_{1}}^{s}$. Moreover, for any $T^{\prime}<T_{1}$, there is a neighborhood $U$ of $\left(u_{0}, f\right)$ in the space $X_{\beta}^{s}$ such that the map $K_{P}$ is analytic from $U$ to the space $\mathcal{Z}_{\beta, T^{\prime}}^{s}$

3. (Global well-posedness) If $s=0$, or $s=1$, or $s \geq 2$, then

$$
H^{s}(S) \times L^{2}\left(-T, T ; H^{s}(S)\right) \subset \mathcal{D}_{s}^{T}\left(K_{P}\right)
$$

The following is a version of Theorem 5.7 for the periodic IVP.

Theorem 6.6 Let $s \geq 0$ and $T>0$ be given. Then, for any $u_{0} \in H^{s+3}(S)$ with $\left[u_{0}\right]=\beta$ and $f \in W^{1,2}\left(-T, T ; H^{s}(S)\right)$ with $[f(\cdot, t)] \equiv 0$, the IVP (6.3) has a unique solution $u \in \mathcal{Z}_{\beta, T}^{s}$. Moreover,

$$
\partial_{t} u \in \mathcal{Z}_{\beta, T}^{s} \quad \text { and } \quad u \in C\left(-T, T ; H^{s+3}(S)\right)
$$

A version of Lemma 4.1 for periodic function classes is the following.

Lemma 6.7 Let $s, \beta \in R$ be given. Then

$$
W^{1 / 2,2}\left(R, H^{s}(S)\right) \subset F_{\beta}^{s^{\prime}}
$$

for any $s^{\prime}<s+3 / 2$.

As a consequence, one can show by methods that are now familiar the following theorem.

Theorem 6.8 Let $s \geq-3 / 2$ be given. For any $u_{0} \in H^{s+\frac{3}{2}}(S)$ with $\left[u_{0}\right]=\beta$ and $f \in W^{1 / 2,2}\left(R, H^{s}(S)\right)$ with $[f(\cdot, t)] \equiv 0$, the corresponding solution $u$ of the IVP (6.3) lies in the space $\mathcal{Z}_{\beta, T}^{s^{\prime}}$ for any $s^{\prime}<s+3 / 2$; where $T>0$ may depend on $\left\|u_{0}\right\|_{s+3 / 2}$ and $\|f\|_{F_{\beta}^{s+3 / 2} \text {. }}$

There are two serious restrictions in the above results. First they require $[f(\cdot, t)] \equiv$ 0 to get an existence result. Secondly, the map $K_{P}$ is only shown to be continuous from the space $X_{\beta}^{s}$ to the space $\mathcal{Z}_{\beta, T}^{s}$ rather than from $H^{s}(S) \times L^{2}\left(R, H^{s}(S)\right)$ to $C\left(-T, T ; H^{s}(S)\right)$. For instance, if $f=0, v_{n}=u_{0} / n$ with $u_{0}$ a non-zero element of $H^{s}(S)$, the above result does not imply that the corresponding solution $u_{n}$ of 
associated IVP (6.3) goes to zero as $n \rightarrow \infty$. These restrictions result from the assumptions entailed in Lemma 6.1. It is not clear whether they can be removed.

On the other hand, it has been known for many years that the map $K$ corresponding to the IVP of the homogeneous $\mathrm{KdV}$ equation is continuous from the space $H^{s}(S)$ to the space $C\left(-T, T ; H^{s}(S)\right)$ when $s>3 / 2$ (cf. [17] and [26]). In the following we show that a similar result holds for the forced KdV equation using energy estimates.

Theorem 6.9 For $s \geq 2$ and $T>0$, if $u_{0} \in H^{s}(S)$ and $f \in L^{1}\left(-T, T ; H^{s}(S)\right)$, then the IVP (6.3) has a unique solution $u \in C\left(-T, T ; H^{s}(S)\right)$. Moreover, the solution $u$ depends continuously on $u_{0}$ in $H^{s}(S)$ and $f$ in $L^{1}\left(-T, T ; H^{s}(S)\right)$.

Remark 6.10 If $3 / 2<s<2$, a local well-posedness result can be obtained using the same approach.

Proof of Theorem 6.9: It suffices to show the existence and continuous dependence. The proof of the uniqueness is a simple exercise for this range of $s$.

First choose a family $\left\{f_{\epsilon}\right\} \subset C^{1}\left(0, T ; H^{\infty}(S)\right)$ and a family $\left\{\phi_{\epsilon}\right\} \subset H^{\infty}(S)$ such that, for any $r \geq 0, \epsilon \geq 0$,

$$
\begin{gathered}
\left\|\phi_{\epsilon}\right\|_{s+r}=O\left(\epsilon^{-\frac{r}{6}}\right), \quad\left\|\phi_{\epsilon}-u_{0}\right\|_{s-r}=o\left(\epsilon^{-\frac{r}{6}}\right), \\
\left\|f_{\epsilon}\right\|_{L^{1}\left(-T, T ; H^{s}(S)\right)}=O\left(\epsilon^{-\frac{r}{6}}\right), \quad \text { and }\left\|f_{\epsilon}-f\right\|_{L^{1}\left(-T, T ; H^{s-r}(S)\right)}=o\left(\epsilon^{\frac{r}{6}}\right)
\end{gathered}
$$

as $\epsilon \downarrow 0$. The construction of such approximating families is straightforward and may be found in [3], for example.

For $\epsilon>0$, consider the regularized IVP

$$
\partial_{t} u^{\epsilon}+u^{\epsilon} \partial_{x} u^{\epsilon}+\partial_{x}^{3} u^{\epsilon}=f_{\epsilon}, \quad u^{\epsilon}(x, 0)=\phi_{\epsilon}(x)
$$

It has a unique solution $u^{\epsilon} \in C\left(-T, T ; H^{\infty}(S)\right)$ (cf. [3] and [26]).

Claim 1. Given $T>0$, there are constants $K_{s}$ independent of $\epsilon$ such that,

$$
\sup _{[-T, T]}\left\|u^{\epsilon}(\cdot, t)\right\|_{s} \leq K_{s}
$$

and for any $r \geq 0$,

$$
\sup _{[-T, T]}\left\|u^{\epsilon}(\cdot, t)\right\|_{s+r}=O\left(\epsilon^{-\frac{r}{6}}\right)
$$

as $\epsilon \downarrow 0$

It is not difficult to show that (6.13) is true when $s=2$ by using the forced version of the conservation laws for the KdV equation. This bound implies $\left\|u^{\epsilon}\right\|_{2}$ is bounded 
by a constant $K_{2}$, independently of $1 \geq \epsilon>0$ and $t \in[-T, T]$. In general, for $l \geq s$, we apply the operator $D^{l}$ to both sides of the equation in (6.12) and take the $L^{2}$-inner product of the resulting equation with $D^{l} u^{\epsilon}$, coming thereby to the relation

$$
\frac{1}{2} \frac{d}{d t}\left\|D^{l} u^{\epsilon}\right\|_{2}^{2}+\left(D^{l}\left(u^{\epsilon} \partial_{x} u^{\epsilon}\right), D^{l} u^{\epsilon}\right)=\left(D^{l} f_{\epsilon}, D^{l} u^{\epsilon}\right) .
$$

Write the second term on the left-hand side of the last equation as

$$
\left(u^{\epsilon} D^{l} \partial_{x} u^{\epsilon}, D^{l} u^{\epsilon}\right)+\left(\left[D^{l}: u^{\epsilon}\right] \partial_{x} u^{\epsilon}, D^{l} u^{\epsilon}\right),
$$

where the commutator $\left[D^{l}: u\right] v=D^{l}(u v)-u D^{l} v$. Applying Lemma 1.1 in [26] and using the fact that $D^{l}$ and $\partial_{x}$ commute shows that, on account of the just mentioned bound on $\left\|u^{\epsilon}\right\|_{2}$,

$$
\begin{aligned}
\left|\left(D^{l}\left(u^{\epsilon} \partial_{x} u^{\epsilon}\right), D^{l} u^{\epsilon}\right)\right| & \leq\left\|u^{\epsilon}\right\| l\left\|u^{\epsilon}\right\|_{2}\left\|D^{l} u^{\epsilon}\right\|+\left|\left(u^{\epsilon} D^{l} \partial_{x} u^{\epsilon}, D^{l} u^{\epsilon}\right)\right| \\
& \leq K_{2}\left\|D^{l} u^{\epsilon}\right\|\left\|u^{\epsilon}\right\|_{l}+\frac{1}{2}\left|\left(\partial_{x} u^{\epsilon} D^{l} u^{\epsilon}, D^{l} u^{\epsilon}\right)\right| \\
& \leq\left(c K_{2}\left(\left\|u^{\epsilon}\right\|+\left\|D^{l} u^{\epsilon}\right\|\right)+\frac{1}{2}\left\|u^{\epsilon}\right\|_{2}\left\|D^{l} u^{\epsilon}\right\|\right)\left\|D^{l} u^{\epsilon}\right\| \\
& \leq c_{1}\left\|D^{l} u^{\epsilon}\right\|+c_{2}\left\|D^{l} u^{\epsilon}\right\|^{2} .
\end{aligned}
$$

In consequence, there appears the differential inequality

$$
\frac{1}{2} \frac{d}{d t}\left\|D^{l} u^{\epsilon}\right\|^{2} \leq c_{2}\left\|D^{l} u^{\epsilon}\right\|^{2}+\left\|D^{l} u^{\epsilon}\right\|\left(\left\|f_{\epsilon}\right\|_{l}+c_{1}\right) .
$$

This in turn implies that

$$
\left\|D^{l} u^{\epsilon}\right\| \leq c\left(\left\|\phi_{\epsilon}\right\|_{l}+\int_{-T}^{T}\left\|f_{\epsilon}\right\|_{l} d t\right)
$$

for a suitable constant $c$ that depends on $T$ and the bound $K_{2}$ on $\left\|u^{\epsilon}\right\|_{2}$. Reference to (6.10) and (6.11) shows that both (6.13) and (6.14) hold.

Next it is shown that $\left\{u^{\epsilon}\right\}_{\epsilon>0}$ is a Cauchy sequence in $C\left(-T, T ; H^{s}(S)\right)$. Its limit as $\epsilon \rightarrow 0$ is the desired solution of the problem. This approach has the advantage that it provides the continuous dependence of solutions on the auxiliary data almost automatically (cf. [3] and [19]).

Assume $0<\epsilon^{\prime}<\epsilon$ and let $w=u^{\epsilon}-u^{\epsilon^{\prime}}$. Then $w$ solves the IVP

$$
\partial_{t} w+u^{\epsilon} \partial_{x} w+w \partial_{x} u^{\epsilon^{\prime}}+\partial_{x}^{3} w=\triangle f, \quad w(x, 0)=\triangle \psi,
$$


where

$$
\triangle f=f_{\epsilon}-f_{\epsilon^{\prime}}, \quad \triangle \psi=\phi_{\epsilon}-\psi_{\epsilon^{\prime}}
$$

\section{Claim 2.}

$$
\sup _{[-T, T]}\|w(\cdot, t)\|_{1}=o\left(\epsilon^{\frac{s-1}{6}}\right)
$$

as $\epsilon \downarrow 0$.

In fact, taking the $L^{2}$-scalar product of each member of the equation in (6.15) with $w$, there appears

$$
\frac{1}{2} \frac{d}{d t}\|w\|^{2}+\left(\partial_{x} u^{\epsilon} w, w\right)-\frac{1}{2}\left(\partial_{x} u^{\epsilon^{\prime}}, w\right)=(\triangle f, w)
$$

which implies that

$$
\begin{aligned}
\sup _{[-T, T]}\|w(\cdot, t)\| & \leq c\left(\|\Delta \psi\|+\int_{-T}^{T}\|\Delta f\| d t\right) \\
& =o\left(\epsilon^{\frac{\varepsilon}{6}}\right)
\end{aligned}
$$

because of (6.13). Then, applying $\partial_{x}$ to each member of the equation in (6.15) and taking the $L^{2}$-scalar product with $\partial_{x} w$, we see after integration by parts that

$$
\begin{aligned}
\frac{d}{d t}\left\|\partial_{x} w\right\|^{2} & =-\left(\left(\partial_{x} u^{\epsilon}+2 \partial_{x} u^{\epsilon^{\prime}}\right) \partial_{x} w, \partial_{x} w\right)-2\left(\partial_{x}^{2} u^{\epsilon^{\prime}} w, \partial_{x} w\right)+2\left(\partial_{x} \triangle f, \partial_{x} w\right) \\
& \leq\left\|u^{\epsilon}+2 u^{\epsilon^{\prime}}\right\|_{2}\left\|\partial_{x} w\right\|^{2}+\left\|u^{\epsilon^{\prime}}\right\|{ }_{2}\|w\|_{\infty}\left\|\partial_{x} w\right\|+\|\Delta f\|\left\|\partial_{x} w\right\| \\
& \leq c_{1}\left\|\partial_{x} w\right\|^{2}+c_{2}\|w\|_{\infty}\left\|\partial_{x} w\right\|+\left\|\partial_{x} \Delta f\right\|\left\|\partial_{x} w\right\|
\end{aligned}
$$

by (6.13), which, when combined with (6.17), implies (6.16).

Now we prove that

$$
\sup _{[-T, T]}\|w(\cdot, t)\|_{s}=o(1)
$$

as $\epsilon \downarrow 0$. Applying $D^{s}$ to each member of the equation in (6.15) and taking the $L^{2}$-scalar product with $D^{s} w$, there obtains the differential equation

$$
\begin{aligned}
\frac{d}{d t}\left\|D^{s} w\right\|^{2} & =-2\left(D^{s}\left(u^{\epsilon} \partial_{x} w\right), D^{s} w\right)-2\left(D^{s}\left(w \partial_{x} u^{\epsilon^{\prime}}\right), D^{s} w\right)+ \\
& +2\left(D^{s} \triangle f, D^{s} w\right) .
\end{aligned}
$$


By again using Lemma 1.1 in [26] and (6.13) in (6.19), it is found as above that

$$
\begin{aligned}
\left|\left(D^{s}\left(u^{\epsilon} \partial_{x} w\right), D^{s} w\right)\right| & =\left|\left(\left[D^{s}: u^{\epsilon}\right] \partial_{x} w, D^{s} w\right)+\left(u^{\epsilon} D^{s+1} w, D^{s} w\right)\right| \\
& \leq c\left\|D^{s} w\right\|^{2}
\end{aligned}
$$

and

$$
\begin{aligned}
\left|\left(D^{s}\left(w \partial_{x} u^{\epsilon^{\prime}}\right), D^{s} w\right)\right| & =\left|\left(\left[D^{s}: w\right] u^{\epsilon^{\prime}}, D^{s} w\right)+\left(w D^{s+1} u^{\epsilon^{\prime}}, D^{s} w\right)\right| \\
& \leq\left\|\left[D^{s}: w\right] u^{\epsilon^{\prime}}\right\|\left\|D^{s} w\right\|+\left\|D^{s+1} u^{\epsilon^{\prime}}\right\|\|w\|_{1}\left\|D^{s} w\right\| \\
& \leq c\left\|D^{s} w\right\|^{2}+o\left(\epsilon^{\frac{s-1}{\epsilon}}\right) O\left(\epsilon^{-\frac{1}{6}}\right)\left\|D^{s} w\right\| \\
& \leq c\left\|D^{s} w\right\|^{2}+o(1)\left\|D^{s} w\right\| .
\end{aligned}
$$

It thus follows from $(6.19)$ that

$$
\frac{d}{d t}\left\|D^{s} w\right\|^{2} \leq c\left\|D^{s} w\right\|^{2}+o(1)\left\|D^{s} w\right\|+\left\|D^{s} \triangle f\right\|\left\|D^{s} w\right\|
$$

which implies (6.18). The proof is complete.

\section{References}

[1] T. R. Akylas, On the excitation of long nonlinear water waves by a moving pressure distribution, J. Fluid Mech. 141 (1984), 455 - 466.

[2] T. B. Benjamin, J. L. Bona and J. J. Mahony, Model equations for long waves in nonlinear, dispersive media, Philos. Trans. Roy. Soc. London A 272 (1972), $47-78$.

[3] J. L. Bona and R. Smith, The initial-value problem for the Korteweg-de Vries equation, Philos. Trans. Roy. Soc. London A 278 (1975), 555 - 601.

[4] J. L. Bona and L. R. Scott, Solutions of the Korteweg-de Vries equations in fractional order Sobolev spaces, Duke Math. J. 43 (1976), 87 - 99.

[5] J. L. Bona, Convergence of periodic wavetrains in the limit of large wavelength, Appl. Scientific Res. 37 (1981), 21 - 30. 
[6] J. Bourgain, Fourier transform restriction phenomena for certain lattice subsets and applications to non-linear evolution equations, part I: Schrödinger equations, Geometric and Functional Analysis 3 (1993), 107 - 156.

[7] J. Bourgain, Fourier transform restriction phenomena for certain lattice subsets and applications to non-linear evolution equations, part II: the KdV equation, Geometric and Functional Analysis 3 (1993), 209 - 262.

[8] A. Cohen, Solutions of the Korteweg-de Vries equation from irregular data, Duke Math. J. 45 (1978), 149 - 181.

[9] S. L. Cole, Transient waves produced by flow past a bump, Wave Motion 7 (1985), 579 - 587.

[10] P. Constantin and J.-C. Saut, Local smoothing properties of dispersive equations, J. Amer. Math. Soc. 1 (1988), 413 - 446.

[11] W. Craig, T. Kappeler and W. A. Strauss, Gain of regularity for equations of the Korteweg-de Vries type, Ann. Inst. Henri Poincaré 9 (1992), 147 - 186.

[12] J. Ginibre and G. Velo, Smoothing properties and retarded estimates for some dispersive evolution equations, preprint.

[13] J. Ginibre and Y. Tsutsumi, Uniqueness for the generalized Korteweg-de Vries equations, SIAM J. Math. Anal. 20 (1989), 1388 - 1425.

[14] R. H. J. Grimshaw and N. Smyth, Resonant flow of a stratified fluid over topography, J. Fluid Mech. 169 (1986), 429 - 464.

[15] T. Kato, Quasilinear equations of evolutions, with applications to partial differential equation, Lecture Notes in Math. 448 (1975), Springer-Verlag, 27 - 50.

[16] T. Kato, On the Korteweg-de Vries equation, Manuscripta Math. 29 (1979), 89 -99 .

[17] T. Kato, On the Cauchy problem for the (generalized) Korteweg-de Vries equations, Advances in Mathematics Supplementary Studies, Studies in Applied Math. 8 (1983), $93-128$.

[18] C. E. Kenig, G. Ponce and L. Vega, Oscillatory integrals and regularity of dispersive equations, Indiana University Math. J. 40 (1991), 33 - 69.

[19] C. E. Kenig, G. Ponce and L. Vega, Well-posedness of the initial value problem for the KdV equation, J. Amer. Math. Soc., 4 (1991), 323 - 347. 
[20] C. E. Kenig, G. Ponce and L. Vega, Well-posedness and scattering results for the generalized Korteweg-de Vries equations via the contraction principle, Comm. Pure Appl. Math. 46 (1993), 527 - 620.

[21] C. E. Kenig, G. Ponce and L. Vega, The Cauchy problem for the Korteweg-de Vries equation in Sobolev spaces of negative indices, Duke Math. J. 71 (1993), 1 -21 .

[22] S. N. Kruzhkov and A. V. Faminskii, Generalized solutions of the Cauchy problem for the Korteweg-de Vries equation, Math. U.S.S.R. Sbornik 48 (1984), 93 138.

[23] S.-J. Lee, Generation of long water waves by moving disturbances, Ph. D. thesis, 1985, California Institute of Technology.

[24] R. M. Miura, The Korteweg-de Vries equation: A survey of results, SIAM review $18(1976), 412-459$.

[25] R. L. Sachs, Classical solutions of the Korteweg-de Vries equation for non-smooth initial data via inverse scattering, Comm. P.D.E. 10 (1985), 29 - 89.

[26] J.-C. Saut and R. Temam, Remarks on the Korteweg-de Vries equation, Israel J. Math. $24(1976), 78-87$.

[27] Y. Tsutsumi, The Cauchy problem for the Korteweg-de Vries equation with measures as initial data, SIAM J. Math. Anal. 20 (1989), 582 - 588.

[28] T. Y. Wu, Generation of upstream advancing solitons by moving disturbances, J. Fluid Mech. 184 (1987), 75 - 99.

[29] B.-Y. Zhang, Some results for the nonlinear dispersive wave equations with applications to control, Ph. D. thesis, University of Wisconsin-Madison, 1990.

[30] B.-Y. Zhang, Taylor series expansion for solutions of the KdV equation with respect to their initial values, the IMA preprint, Series \# 1015 Aug. 1992 (to appear in J. Funct. Anal.).

[31] B.-Y. Zhang, A remark on the Cauchy problem for the Korteweg-de Vries equation on a periodic domain, the IMA preprint, Series \# 1155 July 1993. 

computation of the real stability radius

Maria Inés Troparevsky, Adaptive control of linear discrete time systems with external disturbances under inaccurate modelling: A case study

Petr Klouček \& Franz S. Rys, Stability of the fractional step $\Theta$-scheme for the nonstationary Navier-Stokes equations

1163

1164

1165

1166

1167

1168

1169

Eduardo Casas, Luis A. Fernández \& Jiongmin Yong, Optimal control of quasilinear parabolic equations

Darrell Duffie, Jin Ma \& Jiongmin Yong, Black's consol rate conjecture

D.G. Aronson \& J.L. Vazquez, Anomalous exponents in nonlinear diffusion

Ruben D. Spies, Local existence and regularity of solutions for a mathematical model of thermomechanical phase transitions in shape memory materials with Landau-Ginzburg free energy

Pu Sun, On circular pipe Poiseuille flow instabilities

Angelo Favini, Mary Ann Horn, Irena Lasiecka \& Daniel Tataru, Global existence, uniqueness and regularity of solutions to a Von Kármán system with nonlinear boundary dissipation

A. Dontchev, Tz. Donchev \& I. Slavov, On the upper semicontinuity of the set of solutions of differential inclusions with a small parameter in the derivative

Jin Ma \& Jiongmin Yong, Regular-singular stochastic controls for higher dimensional diffusions - dynamic programming approach

Alex Solomonoff, Bayes finite difference schemes

Todd Arbogast \& Zhangxin Chen, On the implementation of mixed methods as nonconforming methods for second order elliptic problems

Zhangxin Chen \& Bernardo Cockburn, Convergence of a finite element method for the drift-diffusion semiconductor device equations: The multidimensional case

Boris Mordukhovich, Optimization and finite difference approximations of nonconvex differential inclusions with free time

Avner Friedman, David S. Ross, and Jianhua Zhang, A Stefan problem for reaction-diffusion system

Alex Solomonoff, Fast algorithms for micromagnetic computations

Nikan B. Firoozye, Homogenization on lattices: Small parameter limits, $H$-measures, and discrete Wigner measures

G. Yin, Adaptive filtering with averaging

Wlodzimierz Byrc and Amir Dembo, Large deviations for quadratic functionals of Gaussian processes

Ilja Schmelzer, 3D anisotropic grid generation with intersection-based geometry interface

Alex Solomonoff, Application of multipole methods to two matrix eigenproblems

A.M. Latypov, Numerical solution of steady euler equations in streamline-aligned orthogonal coordinates

Bei Hu \& Hong-Ming Yin, Semilinear parabolic equations with prescribed energy

Bei Hu \& Jianhua Zhang, Global existence for a class of Non-Fickian polymer-penetrant systems

Rongze Zhao \& Thomas A. Posbergh, Robust stabilization of a uniformly rotating rigid body

Mary Ann Horn \& Irena Lasiecka, Uniform decay of weak solutions to a von Kármán plate with nonlinear boundary dissipation

Mary Ann Horn, Irena Lasiecka \& Daniel Tataru, Well-posedness and uniform decay rates for weak solutions to a von Kármán system with nonlinear dissipative boundary conditions

Mary Ann Horn, Nonlinear boundary stabilization of a von Kármán plate via bending moments only

Frank H. Shaw \& Charles J. Geyer, Constrained covariance component models

Tomasz Luczaka, A greedy algorithm estimating the height of random trees

Timo Seppäläinen, Maximum entropy principles for disordered spins

Yuandan Lin, Eduardo D. Sontag \& Yuan Wang, Recent results on Lyapunov-theoretic techniques for nonlinear stability

Svante Janson, Random regular graphs: Asymptotic distributions and contiguity

Rachid Ababou, Random porous media flow on large 3-D grids: Numerics, performance, \& application to homogenization

Moshe Fridman, Hidden Markov model regression

Petr Klouček, Bo Li \& Mitchell Luskin, Analysis of a class of nonconforming finite elements for Crystalline microstructures

Steven P. Lalley, Random series in inverse Pisot powers

Rudy Yaksick, Expected optimal exercise time of a perpetual American option: A closed-form solution

Rudy Yaksick, Valuation of an American put catastrophe insurance futures option: A Martingale approach

János Pach, Farhad Shahrokhi \& Mario Szegedy, Application of the crossing number

Avner Friedman \& Chaocheng Huang, Averaged motion of charged particles under their self-induced electric

Joel Spencer, The Erdös-Hanani conjecture via Talagrand's inequality 
Zhangxin Chen, Superconvergence results for Galerkin methods for wave propagation in various porous media

Russell Lyons, Robin Pemantle \& Yuval Peres, When does a branching process grow like its mean? Conceptual proofs of $L \log L$ criteria

Robin Pemantle, Maximum variation of total risk

Robin Pemantle \& Yuval Peres, Galton-Watson trees with the same mean have the same polar sets

Robin Pemantle, A shuffle that mixes sets of any fixed size much faster than it mixes the whole deck

Itai Benjamini, Robin Pemantle \& Yuval Peres, Martin capacity for Markov chains and random walks in varying dimensions

Wlodzimierz Bryc \& Amir Dembo, On large deviations of empirical measures for stationary Gaussian processes Martin Hildebrand, Some random processes related to affine random walks

Alexander E. Mazel \& Yurii M. Suhov, Ground states of a Boson quantum lattice model

Roger L. Fosdick \& Darren E. Mason, Single phase energy minimizers for materials with nonlocal spatial dependence

Bruce Hajek, Load balancing in infinite networks

Petr Klouček, The transonic flow problems stability analysis and numerical results

Petr Klouček, On the existence of the entropic solutions for the transonic flow problem

David A. Schmidt \& Chjan C. Lim, Full sign-invertibility and symplectic matrices

Piermarco Cannarsa \& Maria Elisabetta Tessitore, Infinite dimensional Hamilton-Jacobi equations and Dirichlet boundary control problems of parabolic type

Zhangxin Chen, Multigrid algorithms for mixed methods for second order elliptic problems

Zhangxin Chen, Expanded mixed finite element methods for linear second order elliptic problems I

Gang Bao, A note on the uniqueness for an inverse diffraction problem

Moshe Fridman, A two state capital asset pricing model

Paolo Baldi, Exact asymptotics for the probability of exit from a domain and applications to simulation

Carl Dou \& Martin Hildebrand, Enumeration and random random walks on finite groups

Jaksa Cvitanic \& Ioannis Karatzas, On portfolio optimization under "drawdown" constraints

Avner Friedman \& Yong Liu, A free boundary problem arising in magnetohydrodynamic system

Dominic Welsh, Randomised approximation schemes for Tutte-Gröthendieck invariants

Zhangxin Chen, Bernardo Cockburn, Carl L. Gardner, \& Joseph W. Jerome. Quantum hydrodynamic simulation of hysteresis in the resonant tunneling diode

E.G. Kalnins, G.C. Williams, \& Willard Miller, Jr., Intrinsic characterisation of the separation constant for spin one and gravitational perturbations in Kerr geometry

Zhangxin Chen, Large-scale averaging analysis of multiphase flow in fractured reservoirs

Bruce Hajek \& Babu Narayanan, Multigraphs with the most edge covers

K.B. Athreya, Entropy maximization

F.I. Karpelevich \& Yu.M. Suhov, Functional equations in the problem of boundedness of stochastic branching dynamics

E. Dibenedetto \& V. Vespri, On the singular equation $\beta(u)_{t}=\Delta u$
M.Ya. Kelbert \& Yu.M. Suhov, The Markov branching random walk and systems of reaction-diffusion (Kolmogorov-Petrovskii-Piskunov) equations

M. Hildebrand, Random walks on random regular simple graphs

W.S. Don \& A. Solomonoff, Accuracy enhancement for higher derivatives using Chebyshev collocation and a mapping technique

D. Gurarie, Symmetries and conservation laws of two-dimensional hydrodynamics

Z. Chen, Finite element methods for the black oil model in petroleum reservoirs

G. Bao \& A. Friedman, Inverse problems for scattering by periodic structure

G. Bao, Some inverse problems in partial differential equations

G. Bao, Diffractive optics in periodic structures: The TM polarization

C.C. $\operatorname{Lim} \&$ D.A. Schmidt, On noneven digraphs and symplectic pairs

H.M. Soner, S.E. Shreve \& J. Cvitanić, There is no nontrivial hedging portfolio for option pricing with transaction costs

D.L. Russell \& B-Yu Zhang, Exact controllability and stabilizability of the Korteweg-de Vries equation

B. Morton, D. Enns \& B-Yu Zhang, Stability of dynamic inversion control laws applied to nonlinear aircraft pitch-axis models

S. Hansen \& G. Weiss, New results on the operator Carleson measure criterion

V.A. Malyshev \& F.M. Spieksma, Intrinsic convergence rate of countable Markov chains

G. Bao, D.C. Dobson \& J.A. Cox, Mathematical studies in rigorous grating theory

G. Bao \& W.W. Symes, On the sensitivity of solutions of hyperbolic equations to the coefficients

D.A. Huntley \& S.H. Davis, Oscillatory and cellular mode coupling in rapid directional solidification

M.J. Donahue. L. Gurvits, C. Darken \& E. Sontag, Rates of convex approximation in non-Hilbert spaces

A. Friedman \& B. Hu, A Stefan problem for multi-dimensional reaction diffusion systems

J.L. Bona \& B-Y. Zhang, The initial-value problem for the forced Korteweg-de Vries equation

1254 